\title{
3D Reconstruction of "In-the-Wild" Faces in Images and Videos
}

\author{
James Booth, Anastasios Roussos, Evangelos Ververas, Epameinondas Anton- \\ akos, Stylianos Ploumpis, Yannis Panagakis, and Stefanos Zafeiriou
}

\begin{abstract}
D Morphable Models (3DMMs) are powerful statistical models of 3D facial shape and texture, and are among the state-of-the-art methods for reconstructing facial shape from single images. With the advent of new 3D sensors, many 3D facial datasets have been collected containing both neutral as well as expressive faces. However, all datasets are captured under controlled conditions. Thus, even though powerful 3D facial shape models can be learnt from such data, it is difficult to build statistical texture models that are sufficient to reconstruct faces captured in unconstrained conditions ("in-the-wild"). In this paper, we propose the first "in-the-wild" 3DMM by combining a statistical model of facial identity and expression shape with an "in-the-wild" texture model. We show that such an approach allows for the development of a greatly simplified fitting procedure for images and videos, as there is no need to optimise with regards to the illumination parameters. We have collected three new benchmarks that combine "in-the-wild" images and video with ground truth 3D facial geometry, the first of their kind, and report extensive quantitative evaluations using them that demonstrate our method is state-of-the-art.
\end{abstract}

Index Terms-3DMM, Morphable Model, RPCA, 3D reconstruction.

\section{INTRODUCTION}

$\mathrm{D}$ URING the past few years, we have witnessed significant improvements in various face analysis tasks such as face detection [1], [2] and 2D facial landmark localisation on static images [3], [4], [5], [6], [7], [8], [9], [10]. This is primarily attributed to the fact that the community has made a considerable effort to collect and annotate facial images captured under unconstrained conditions [11], [12], [13], [14], [15] (commonly referred to as "in-the-wild") and to develop discriminative methodologies that can capitalise on the availability of such a large amount of data. Nevertheless, discriminative techniques cannot be applied for 3D facial shape reconstruction "in-the-wild", due to lack of groundtruth data.

3D facial shape reconstruction from a single image or a video captured under "in-the-wild" conditions is still an open and challenging problem in Computer Vision. This is mainly due to the fact that the general problem of extracting the 3D facial shape from a single image, or even a video sequence, is an ill-posed problem which is notoriously difficult to solve without the use of any statistical priors for the shape and texture of faces. That is, without prior knowledge regarding the shape of the object athand there are inherent ambiguities present in the problem. The pixel intensity at a location in an image is the result of a complex combination of the underlying shape of the object, the surface albedo and reflectance characteristics, camera parameters and the arrangement of scene lighting and other objects in the scene. Hence, there are potentially infinite solutions to the problem.

Furthermore, learning statistical priors of 3D facial shape and texture for "in-the-wild" images is challenging even with

- The authors are with the Department of Computing, Imperial College London, South Kensington Campus, London SW7 2AZ, UK.

- A. Roussos is also with the College of Engineering, Mathematics and Physical Sciences, University of Exeter, UK.

- Y. Panagakis is also with the Department of Computer Science, Middlesex University London.

E-mails: see https://ibug.doc.ic.ac.uk/people modern 3D acquisition devices, as, even though there has been a considerable improvement in the performance of such scanners, they still cannot operate in arbitrary conditions. As a result of these restrictions, all current 3D facial databases have been captured in controlled conditions.

With the available 3D facial data, it is feasible to learn a powerful statistical model of the facial shape that generalises well for both identity and expression [16], [17], [18]. However, it is not possible to construct a statistical model of the facial texture that generalises well for "in-the-wild" images and is, at the same time, in correspondence with the statistical shape model. That is the reason why current state-of-the-art $3 \mathrm{D}$ face reconstruction methodologies rely solely on fitting a statistical 3D facial shape prior on a sparse set of landmarks [19], [20].

In this paper, we make a number of contributions that enable the use of 3DMMs for "in-the-wild" face reconstruction (Fig. 1):

- Motivated by the success of feature-based (e.g., HOG [21], SIFT [22]) Active Appearance Models (AAMs) [8], [23], we propose a methodology for learning a statistical texture model from "in-the-wild" facial images, which is in full correspondence with a statistical shape prior that exhibits both identity and expression variations.

- By capitalising on the recent advancements in fitting statistical deformable models [8], [24], [25], [26], we propose a novel and fast algorithm for fitting our "inthe-wild" 3DMMs on images and videos. We show that the advantage of using the "in-the-wild" feature-based texture model is that the fitting strategy can be significantly simplified since there is no need to optimise with respect to illumination parameters.

- We make the implementation of our algorithm publicly available $^{1}$ as part of the Menpo Project [27]. We strongly believe that this can be of great benefit to the research

1. https://ibug.doc.ic.ac.uk/resources/itwmm 

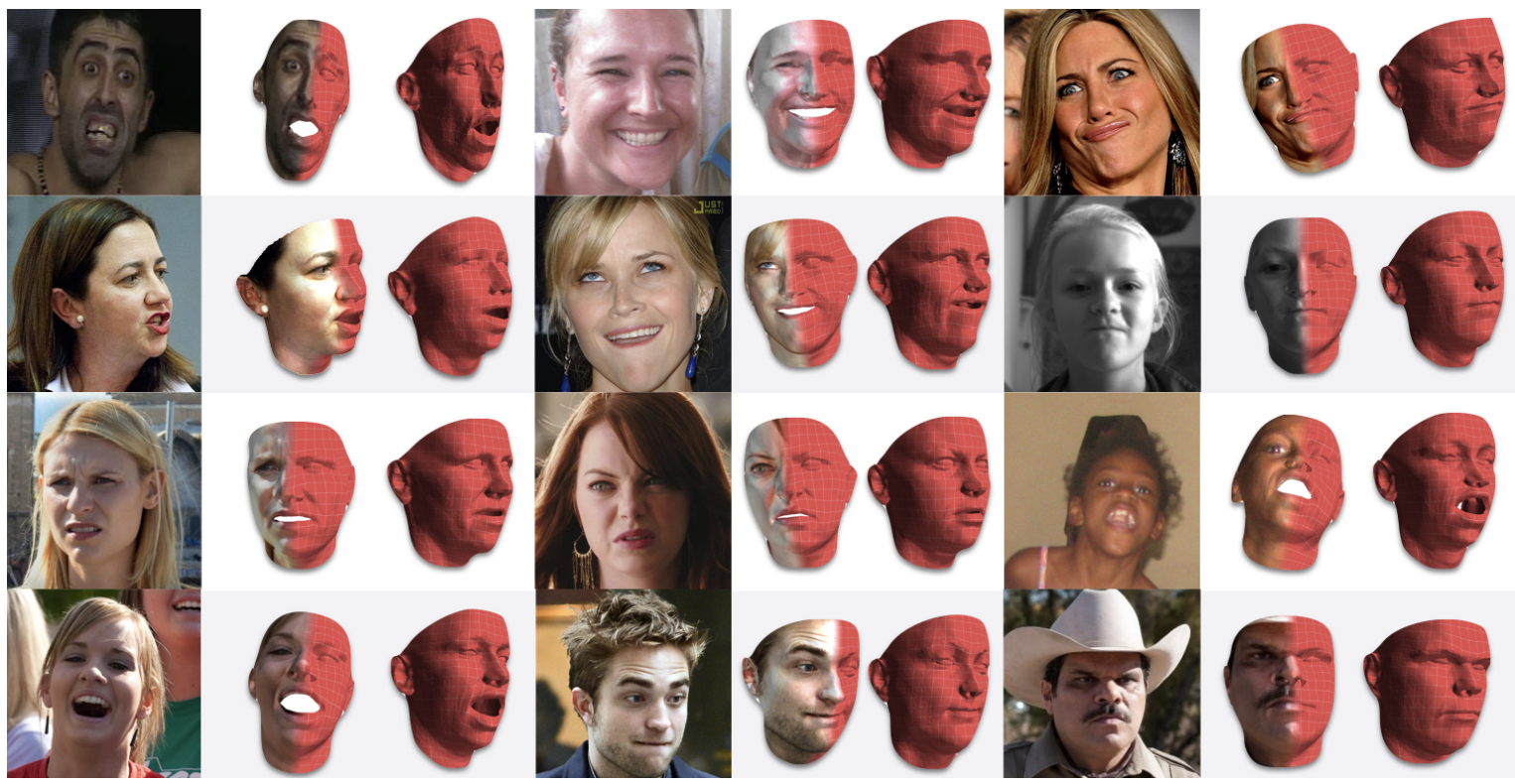

Fig. 1. Results of our 3DMM image fitting method ITW(Basel) on "in-the-wild" images from the 300W dataset [15]. We note that our proposed technique is able to handle extremely challenging pose, illumination, and expression variations, returning plausible $3 \mathrm{D}$ facial shapes in all the above cases.

community, given the lack of robust open-source implementations for fitting 3DMMs.

- In order to provide quantitative evaluations we collect three new benchmarks which couple images and videos of faces with 3D ground truth shape information - KF-ITW, 4DMaja and 3dMDLab. For the benefit of the research community, we make the benchmarks publicly available too ${ }^{1}$.

- We present extensive quantitative and qualitative evaluations of our proposed method against a wide range of state-of-the-art alternatives, which demonstrates the clear merits of our technique. [18].

The remainder of the paper is structured as follows. In Sec. 2 we briefly outline the background on face reconstruction from monocular cameras. In Sec. 3 we elaborate on the construction of our "in-the-wild" 3DMM, whilst in Sec. 4 we outline the proposed optimisation for fitting "in-the-wild" images (Sec. 4.1) and videos (Sec. 4.2) with our model. Sec. 5 describes our three new benchmarks, the first of their kind, which provide "in-the-wild" images and video sequences with ground-truth 3D facial shape. We outline a series of quantitative and qualitative experiments in Sec. 6, and end with conclusions in Sec. 7.

\section{BACKGROUND}

Accurate recovery of the true 3D structure of a scene captured by an image or video is arguably one of the core problems in computer vision. Although it is feasible to recover many properties of a scene's background, the geometry of the objects within the scene is the most important task, since it enables the acquisition of powerful and descriptive models from which to perform inference. In particular, the 3D shape of the underlying objects is arguably the strongest cue for common tasks such as object recognition and localisation. However, the general problem of recovering the 3D shape of an object from a single image, or even a set of images with different viewpoints, is ill-conditioned. Even when provided with multiple images, additional information about the scene or details about the capturing conditions, 3D shape recovery is full of ambiguities. Many strategies have been proposed for solving this problem.

In contrast to the difficulty of the general case, recovery of 3D facial shape has been successful in scenarios with controlled recording conditions. Human faces exhibit several characteristics that are beneficial for performing shape recovery: $(i)$ they have approximately homogeneous configuration (all healthy human faces have the same parts, such as eyes, nose and mouth, in the same approximate locations), (ii) they have convex shape, and (iii) they exhibit approximately Lambertian reflectance [28], [29], [30], [31], [32], [33], [34], [35]. Nevertheless, the task is still very challenging since faces are highly deformable; their appearance changes dramatically depending on the illumination conditions and can exhibit severe self-occlusions depending on the viewpoint.

In this paper, we are interested in the very challenging problem of 3D face reconstruction from still images or videos captured under unconstrained conditions, i.e. "in-the-wild". Hence, we herein review methodologies that do not require the use of any specialised machinery (e.g., depth or stereo cameras).

Although the relevant literature is very extensive, most works can be broadly categorized as follows:

Shape-from-Shading (SfS): These methods expect a single image [36] (or a collection of images) as input and use image formation assumptions (usually the Lambertian reflectance assumption) to recover surface shape. There is considerable research in SfS for generic surfaces, as well as faces [36], [37], [38], [39], [40], [41], [42]. However, generic SfS techniques do not produce very convincing results for faces [39], unless face shape priors are introduced [36], [38] or SfS is jointly performed in a large collection of facial images [41], [42]. The current state-ofthe-art techniques include methods such as [41], [42], which are able to recover some facial details. However, they require dense alignment to be performed (e.g., by using elaborate optical flow techniques [42]) and they are only suitable for recovering $2.5 \mathrm{D}$ 
information and not full 3D shape.

3D Morphable Models (3DMM): The 3DMM fitting proposed in the work of Blanz \& Vetter [43], [44] was among the first model-based 3D facial recovery approaches. The first 3DMM was built using 200 faces captured in well-controlled conditions displaying only the neutral expression. That is the reason why the method was only shown to work on real-world, but not "in-thewild", images. Since then, many extensions have been proposed to the original method [45], [46], [47], [48]. Although model-based SfS may also consider similarity to a facial model as a measure of reconstruction accuracy, 3DMMs are unique in explicitly rendering images of faces for the purpose of 3D recovery. Until recently, due to the lack of available texture models, 3DMMs were deemed suitable only for images captured under controlled conditions. Hence, many works considered only fitting a dense shape model to a collection of sparse landmarks that were localised in the image [19], [20]. In this paper, we make a significant step further and demonstrate how to train the first "in-the-wild" 3DMM.

Structure-from-Motion (SfM): These methods employ geometric constraints in order to recover 3D structure across multiple images or frames of a sequence. Although the majority of research in this area is not face specific, facial data is commonly used to demonstrate the effectiveness of a method [49]. Nevertheless, the lack of use of appropriate facial shape models makes the problem of dense 3D face reconstruction very difficult to solve. This is due to the fact that the dense SfM requires the solution of a very high dimensional non-convex optimisation problem [49] which assumes the presence of very accurate dense flow [50], restrictions that make such techniques applicable mainly in controlled recording conditions [49]. Nevertheless, similarly to [51], sparse SfM applied on a collection of tracked landmarks can be used to provide an initialisation to our methodology when it comes to reconstructing faces in videos.

\section{Model Training}

A 3DMM consists of three parametric models: the shape, camera and texture models.

\subsection{Shape Modelling}

Let us denote the 3D mesh (shape) of an object with $N$ vertices as a $3 N \times 1$ vector

$$
\mathbf{s}=\left[\mathbf{x}_{1}^{\top}, \ldots, \mathbf{x}_{N}^{\top}\right]^{\top}=\left[x_{1}, y_{1}, z_{1}, \ldots, x_{N}, y_{N}, z_{N}\right]^{\top}
$$

where $\mathbf{x}_{i}=\left[x_{i}, y_{i}, z_{i}\right]^{\top}$ are the object-centered Cartesian coordinates of the $i$-th vertex.

We first of all consider an identity shape model, i.e. a model of shape variation across different individuals, assuming that all shapes are under neutral expression. For this, we adopt the recently released LSFM model [18], [52], the largest-scale 3D Morphable Model (3DMM) of facial identity which was built from around 10,000 scans of different individuals.

A 3D shape model like the one in LSFM is constructed by first bringing a set of 3D training meshes into dense correspondence so that each is described with the same number of vertices and all samples have a shared semantic ordering. The corresponded meshes, $\left\{\mathbf{s}_{i}\right\}$, are then brought into a shape space by applying Generalised Procrustes Analysis and then Principal Component Analysis (PCA) is performed which results in $\left\{\overline{\mathbf{s}}_{i d}, \mathbf{U}_{i d}, \boldsymbol{\Sigma}_{i d}\right\}$, where $\overline{\mathbf{s}}_{i d} \in \mathbb{R}^{3 N}$ is the mean shape vector, $\mathbf{U}_{i d} \in \mathbb{R}^{3 N \times n_{p}}$

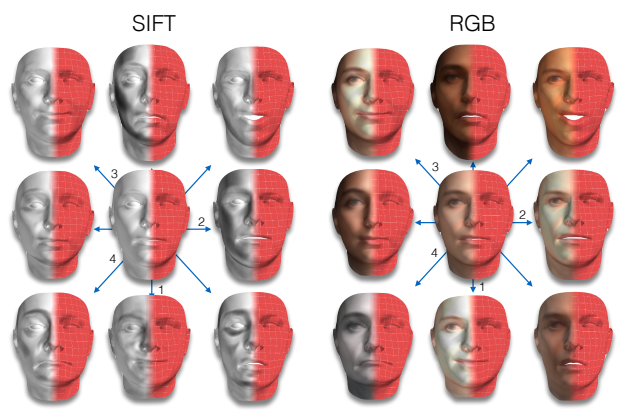

Fig. 2. Left: The mean and first four shape and SIFT texture principal components of our "in-the-wild" SIFT texture model. Right: To aid in interpretation we also show the equivalent RGB basis.

is the orthonormal basis after keeping the first $n_{p}$ principal components and $\boldsymbol{\Sigma}_{i d} \in \mathbb{R}^{n_{p} \times n_{p}}$ is a diagonal matrix with the standard deviations of the corresponding principal components. Let $\widetilde{\mathbf{U}}_{i d}=\mathbf{U}_{i d} \boldsymbol{\Sigma}_{i d}$ be the identity basis with basis vectors that have absorbed the standard deviation of the corresponding mode of variation so that the shape parameters $\mathbf{p}=\left[p_{1}, \ldots, p_{n_{p}}\right]^{\top}$ are normalised to have unit variance. Therefore, assuming normal prior distributions, we have $\mathbf{p} \sim \mathcal{N}\left(\mathbf{0}, \mathbf{I}_{n_{p}}\right)$, where $\mathbf{I}_{n}$ denotes the $n \times n$ identity matrix. Also, a 3D shape instance of a novel identity can be generated using this model as a function of the parameters p:

$$
\mathcal{S}_{i d}(\mathbf{p})=\overline{\mathbf{s}}_{i d}+\widetilde{\mathbf{U}}_{i d} \mathbf{p}
$$

Visualisations of the identity model are included in the Supplementary Material.

Furthermore, we also consider a 3D shape model of expression variations, as offsets from a given identity shape $\mathcal{S}_{i d}$. For this we use the blendshapes model of Facewarehouse [16]. We adopt Nonrigid ICP [53] to accurately register this model with the LSFM identity model. After this procedure, the expression model can be represented with the triplet $\left\{\overline{\mathbf{s}}_{\text {exp }}, \mathbf{U}_{\text {exp }}, \boldsymbol{\Sigma}_{\text {exp }}\right\}$, where $\overline{\mathbf{s}}_{\text {exp }} \in \mathbb{R}^{3 N}$ is the mean expression offset, $\mathbf{U}_{\text {exp }} \in \mathbb{R}^{3 N \times n_{q}}$ is the orthonormal expression basis having $n_{q}$ principal components and $\boldsymbol{\Sigma}_{\text {exp }} \in \mathbb{R}^{n_{q} \times n_{q}}$ is the diagonal matrix with the corresponding standard deviations. Similarly with the identity model, we consider the basis $\widetilde{\mathbf{U}}_{\exp }=\mathbf{U}_{\exp } \boldsymbol{\Sigma}_{\text {exp }}$ and the associated normalised parameters $\mathbf{q} \sim \mathcal{N}\left(\mathbf{0}, \mathbf{I}_{n_{q}}\right)$.

Combining the two aforementioned models, we end up with the following combined model that represents the 3D facial shape of any identity under any expression (please refer to the Supplementary Material for a visualisation):

$$
\mathcal{S}(\mathbf{p}, \mathbf{q})=\overline{\mathbf{s}}+\widetilde{\mathbf{U}}_{i d} \mathbf{p}+\widetilde{\mathbf{U}}_{e x p} \mathbf{q}
$$

where $\overline{\mathbf{s}}=\overline{\mathbf{s}}_{i d}+\overline{\mathbf{s}}_{\text {exp }}$ is the overall mean shape, $\mathbf{p}$ is the vector with the identity parameters and $\mathbf{q}$ is the vector with the expression parameters.

\subsection{Camera Model}

The purpose of the camera model is to map (project) the objectcentred Cartesian coordinates of a 3D mesh instance $\mathbf{s}$ into 2D Cartesian coordinates on an image plane.

The projection of a $3 \mathrm{D}$ point $\mathbf{x}=[x, y, z]^{\top}$ into its $2 \mathrm{D}$ location in the image plane $\mathbf{x}^{\prime}=\left[x^{\prime}, y^{\prime}\right]^{\top}$ involves two steps. 
First, the 3D point is rotated and translated using a linear view transformation to bring it in the camera reference frame:

$$
\mathbf{v}=\left[v_{x}, v_{y}, v_{z}\right]^{\top}=\mathbf{R}_{v} \mathbf{x}+\mathbf{t}_{v}
$$

where $\mathbf{R}_{v} \in \mathbb{R}^{3 \times 3}$ and $\mathbf{t}_{v}=\left[t_{x}, t_{y}, t_{z}\right]^{\top}$ are the camera's 3D rotation and translation components, respectively. This is based on the fact that, without loss of generality, we can assume that the observed facial shape is still and that the relative change in 3D pose between camera and object is only due to camera motion.

Then, a camera projection is applied as:

$$
\mathbf{x}^{\prime}=\pi\left(\mathbf{c}_{\text {intr }}, \mathbf{v}\right)
$$

where $\mathbf{c}_{\text {intr }}$ is a vector with the camera's intrinsic parameters.

The above generic formulation can be applied to any camera model. For example, in the case of a perspective camera with its principal point fixed at the image centre, $\mathbf{c}_{\text {intr }}=\phi$, where $\phi$ is the focal length and the camera projection function is defined as:

$$
\pi(\mathbf{v}, \phi)=\frac{\phi}{v_{z}}\left[\begin{array}{l}
v_{x} \\
v_{y}
\end{array}\right]+\left[\begin{array}{l}
c_{x} \\
c_{y}
\end{array}\right]
$$

where $\left[c_{x}, c_{y}\right]^{\top}$ are the image coordinates of the image centre.

In the case of a scaled orthographic camera projection, $\mathbf{c}_{\text {intr }}=$ $\sigma$, where $\sigma$ is the scale parameter of the camera and the camera projection function is given by:

$$
\pi(\mathbf{v}, \sigma)=\sigma\left[\begin{array}{l}
v_{x} \\
v_{y}
\end{array}\right]
$$

Quaternions. We parametrise the 3D rotation with quaternions [54], [55]. The quaternion uses four parameters $\mathbf{q}=$ $\left[q_{0}, q_{1}, q_{2}, q_{3}\right]^{\mathrm{\top}}$ in order to express a $3 \mathrm{D}$ rotation as

$$
\mathbf{R}_{v}=2\left[\begin{array}{ccc}
\frac{1}{2}-q_{2}^{2}-q_{3}^{2} & q_{1} q_{2}-q_{0} q_{3} & q_{1} q_{3}+q_{0} q_{2} \\
q_{1} q_{2}+q_{0} q_{3} & \frac{1}{2}-q_{1}^{2}-q_{3}^{2} & q_{2} q_{3}-q_{0} q_{1} \\
q_{1} q_{3}-q_{0} q_{2} & q_{2} q_{3}+q_{0} q_{1} & \frac{1}{2}-q_{1}^{2}-q_{2}^{2}
\end{array}\right]
$$

Note that by enforcing a unit norm constraint on the quaternion vector, i.e. $\mathbf{q}^{\top} \mathbf{q}=1$, the rotation matrix constraints of orthogonality with unit determinant are withheld. Given the unit norm property, the quaternion can be seen as a three-parameter vector $\left[q_{1}, q_{2}, q_{3}\right]^{\top}$ and a scalar $q_{0}=\sqrt{1-q_{1}^{2}-q_{2}^{2}-q_{3}^{2}}$. Most existing works on $3 \mathrm{DMM}$ parametrise the rotation matrix $\mathbf{R}_{v}$ using the three Euler angles that define the rotations around the horizontal, vertical and camera axes. Even thought Euler angles are more naturally interpretable, they have strong disadvantages when employed within an optimisation procedure, most notably the solution ambiguity and the gimbal lock effect.

Camera function. The projection operation performed by the camera model of the 3DMM can be expressed with the function $\mathcal{P}(\mathbf{s}, \mathbf{c}): \mathbb{R}^{3 N} \rightarrow \mathbb{R}^{2 N}$, which applies the transformations of Eqs. (4) and (6) on the points of provided 3D mesh $\mathbf{s}$ with

$$
\mathbf{c}=\left[\mathbf{c}_{\text {intr }}, q_{1}, q_{2}, q_{3}, t_{x}, t_{y}, t_{z}\right]^{\top}
$$

being the vector of camera parameters with length $n_{c}=7$. For abbreviation purposes, we represent the camera model of the $3 \mathrm{DMM}$ with the function $\mathcal{W}: \mathbb{R}^{n_{p}, n_{c}} \rightarrow \mathbb{R}^{2 N}$ as

$$
\mathcal{W}(\mathbf{p}, \mathbf{q}, \mathbf{c}) \equiv \mathcal{P}(\mathcal{S}(\mathbf{p}, \mathbf{q}), \mathbf{c})
$$

where $\mathcal{S}(\mathbf{p}, \mathbf{q})$ is a 3D mesh instance using Eq. (2).

\subsection{Feature-Based Texture Model}

A key component of our proposed 3DMM is the generation of feature-based "in-the-wild" texture models which allow us to avoid the estimation of illumination parameters in our fitting method. To construct such models, it would not be effective to use the texture from 3D facial scans, as usually done in the construction of 3DMMs [18], [43], [52], since the illumination conditions are excessively controlled in such scans. On the contrary, our goal is to model the texture of faces, as captured by images and videos under completely uncontrolled conditions. Therefore, we utilise a large collection of "in-the-wild" facial images, accompanied with a sparse set of facial landmarks.

We assume that for the aforementioned set of $M$ "in-thewild" images $\left\{\mathbf{I}_{i}\right\}_{1}^{M}$, we have access to the associated camera and shape parameters $\left\{\mathbf{p}_{\mathbf{i}}, \mathbf{q}_{\mathbf{i}}, \mathbf{c}_{\mathbf{i}}\right\}$. These parameters are initially estimated by fitting the combined 3D shape model on the sparse 2D landmarks. Let us also define a dense feature extraction function

$$
\mathcal{F}: \mathbb{R}^{H \times W \times N_{\text {colors }}} \rightarrow \mathbb{R}^{H \times W \times C}
$$

where $H, W, N_{\text {colors }}$ are the width, height and number of color channels respectively of the input image and $C$ is the number of channels of the feature-based image. For each image, we first compute its feature-based representation as $\mathbf{F}_{i}=\mathcal{F}\left(\mathbf{I}_{i}\right)$ and then use Eq. (10) to sample it at each vertex location to build back a vectorised texture sample $\mathbf{t}_{i}=\mathbf{F}_{i}\left(\mathcal{W}\left(\mathbf{p}_{\mathbf{i}}, \mathbf{q}_{\mathbf{i}}, \mathbf{c}_{\mathbf{i}}\right)\right) \in \mathbb{R}^{C N}$. This texture sample will be nonsensical for some regions mainly due to self-occlusions present in the mesh projected in the image space $\mathcal{W}\left(\mathbf{p}_{\mathbf{i}}, \mathbf{q}_{\mathbf{i}}, \mathbf{c}_{\mathbf{i}}\right)$. To alleviate these issues, we cast a ray from the camera to each vertex and test for self-intersections with the triangulation of the mesh in order to learn a per-vertex occlusion mask $\mathbf{m}_{i} \in \mathbb{R}^{N}$ for the projected sample.

Let us create the matrix $\mathbf{X}=\left[\mathbf{t}_{1}, \ldots, \mathbf{t}_{M}\right] \in \mathbb{R}^{C N \times M}$ by concatenating the $M$ grossly corrupted feature-based texture vectors with missing entries that are represented by the masks $\mathbf{m}_{i}$. To robustly build a texture model based on this incomplete data, we need to recover a low-rank matrix $\mathbf{L} \in \mathbb{R}^{C N \times M}$ representing the clean facial texture and a sparse matrix $\mathbf{E} \in \mathbb{R}^{C N \times M}$ accounting for gross but sparse non-Gaussian noise such that $\mathbf{X}=\mathbf{L}+\mathbf{E}$. To simultaneously recover both $\mathbf{L}$ and $\mathbf{E}$ from incomplete and grossly corrupted observations, the Principal Component Pursuit with missing values problem [56] is solved

$$
\begin{aligned}
& \underset{\mathbf{L}, \mathbf{E}}{\arg \min }\|\mathbf{L}\|_{*}+\lambda\|\mathbf{E}\|_{1} \\
& \text { s.t. } \mathcal{P}_{\Omega}(\mathbf{X})=\mathcal{P}_{\Omega}(\mathbf{L}+\mathbf{E}) \text {, }
\end{aligned}
$$

where $\|\cdot\|_{*}$ denotes the nuclear norm, $\|\cdot\|_{1}$ is the matrix $\ell_{1}$ norm and $\lambda>0$ is a regularizer. $\Omega$ represents the set of locations corresponding to the observed entries of $\mathbf{X}$ (i.e., $(i, j) \in \Omega$ if $\left.m_{i}=m_{j}=1\right)$. Then, $\mathcal{P}_{\Omega}(\mathbf{X})$ is defined as the projection of the matrix $\mathbf{X}$ on the observed entries $\Omega$, namely $\mathcal{P}_{\Omega}(\mathbf{X})_{i j}=x_{i j}$ if $(i, j) \in \Omega$ and $\mathcal{P}_{\Omega}(\mathbf{X})_{i j}=0$ otherwise. The unique solution of the convex optimization problem in Eq. (12) is found by employing an Alternating Direction Method of Multipliers-based algorithm [57].

The final texture model is created by applying PCA on $\mathbf{L}$ (the set of reconstructed feature-based textures acquired from the previous procedure). This results in $\left\{\overline{\mathbf{t}}, \mathbf{U}_{t}\right\}$, where $\overline{\mathbf{t}} \in \mathbb{R}^{C N}$ is the mean texture vector and $\mathbf{U}_{t} \in \mathbb{R}^{C N \times n_{t}}$ is the orthonormal basis after keeping the first $n_{t}$ principal components. This model 


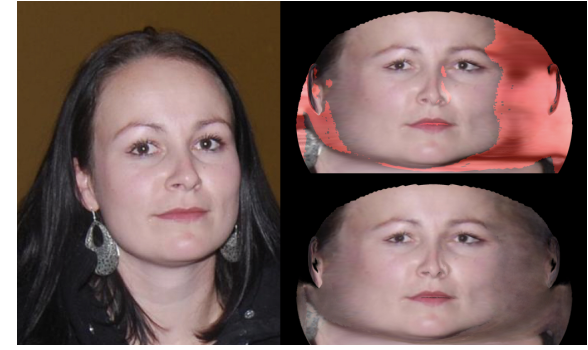

Fig. 3. Building an ITW texture model. The red coloured region denotes the occlusion mask obtained by fitting the 3D shape model on the sparse 2D landmarks of the original image.

can be used to generate novel 3D feature-based texture instances with the function $\mathcal{T}: \mathbb{R}^{n_{t}} \rightarrow \mathbb{R}^{C N}$ as

$$
\mathcal{T}(\boldsymbol{\lambda})=\overline{\mathbf{t}}+\mathbf{U}_{t} \boldsymbol{\lambda}
$$

where $\boldsymbol{\lambda}=\left[\lambda_{1}, \ldots, \lambda_{n_{t}}\right]^{\top}$ are the $n_{t}$ texture parameters.

Finally, an iterative procedure can be employed in order to refine the texture. Starting with the $3 \mathrm{D}$ fits provided by using only the 2D landmarks [58] a texture model can be learnt using the above procedure. This texture model can then be used with the proposed 3DMM fitting algorithm on the same data to refine the quality of the 3D fits, allowing for the recovery of an improved texture model. This could be repeated over multiple iterations, but we have empirically found that a single refinement iteration is often adequate. In the case of single-image fitting, this procedure is done in a separate training phase, which needs to be performed only once. In the case of video fitting, this can be done for every input video.

\section{Model Fitting}

We now present a Gauss-Newton-style energy minimisation to fit our "in-the-wild" 3DMM. First we consider the case of fitting single images, then we proceed to consider fitting video sequences. Please note that we make the source code of our model fitting method publicly available.

\subsection{Fitting on Single Images}

\subsubsection{Proposed Energy Formulation}

To fit the 3DMM on single images, we propose to minimise the following cost function:

$$
\begin{aligned}
E(\mathbf{p}, \mathbf{q}, \mathbf{c}, \boldsymbol{\lambda}) & =E_{\text {text }}(\mathbf{p}, \mathbf{q}, \mathbf{c}, \boldsymbol{\lambda})+c_{\ell} E_{\text {land }}(\mathbf{p}, \mathbf{q}, \mathbf{c}) \\
& +E_{\text {priors }}(\mathbf{p}, \mathbf{q})
\end{aligned}
$$

where $E_{\text {text }}$ is a texture reconstruction term, $E_{\text {land }}$ is a sparse 2D landmarks term and $E_{\text {priors }}$ is a shape priors term that regularises the shape parameters. Also $c_{\ell}$ is the balancing weight of the $E_{\text {land }}$ term. The energy $E$ depends on the shape $(\mathbf{p}, \mathbf{q})$, texture $\boldsymbol{\lambda}$ and camera $\mathbf{c}$ parameters and these are the quantities that we seek to estimate by minimising it. The terms $E_{\text {land }}$ and $E_{\text {priors }}$ are optional and aim to facilitate the optimisation procedure in order to converge faster and to a better minimum. Note that thanks to the proposed "in-the-wild" feature-based texture model, the cost function does not include any parametric illumination model similar to the ones in the related literature [43], [44], which greatly simplifies the optimisation. Next, we present every term of the energy.
The texture reconstruction term $\left(E_{\text {text }}\right)$ is the main data term of the optimisation problem. It depends on shape, texture and camera parameters and penalises the squared $L^{2}$ norm of the difference between the image feature-based texture that corresponds to the projected $2 \mathrm{D}$ locations of the $3 \mathrm{D}$ shape instance and the texture instance of the 3DMM:

$$
E_{\text {text }}(\mathbf{p}, \mathbf{q}, \mathbf{c}, \boldsymbol{\lambda})=\|\mathbf{F}(\mathcal{W}(\mathbf{p}, \mathbf{q}, \mathbf{c}))-\mathcal{T}(\boldsymbol{\lambda})\|^{2}
$$

where $\mathbf{F}=\mathcal{F}(\mathbf{I})$ denotes the feature-based representation with $C$ channels of an input image $\mathbf{I}$ using Eq. (11). Note that $\mathbf{F}(\mathcal{W}(\mathbf{p}, \mathbf{q}, \mathbf{c})) \in \mathbb{R}^{C N}$ denotes the operation of sampling the feature-based input image on the projected 2D locations of the 3D shape instance acquired by the camera model (Eq. (10)).

The 2D landmarks term ( $\left.E_{\text {land }}\right)$ is an auxiliary data term that is based on sparse 2D landmarks:

$$
E_{\text {land }}(\mathbf{p}, \mathbf{q}, \mathbf{c})=\left\|\mathcal{W}_{l}(\mathbf{p}, \mathbf{q}, \mathbf{c})-\ell\right\|^{2}
$$

where $\boldsymbol{\ell}=\left[x_{1}, y_{1}, \ldots, x_{L}, y_{L}\right]^{\top}$ denotes a set of $L$ sparse 2D landmark points $(L \ll N)$ defined on the image coordinate system and $\mathcal{W}_{l}(\mathbf{p}, \mathbf{q}, \mathbf{c})$ returns the $2 L \times 1$ vector of $2 \mathrm{D}$ projected locations of these $L$ sparse landmarks. Intuitively, this term aims to drive the optimisation procedure using the selected sparse landmarks as anchors for which we have the optimal locations $\ell$. In this way, the camera parameters can be rapidly adapted.

The shape priors term ( $E_{\text {priors }}$ ) aims to avoid over-fitting effects by penalizing reconstructed faces that are statistically unlikely given the parametric shape model. It consists of two optional prior terms over the identity and expression parameters, $\mathbf{p}$ and $\mathbf{q}$. Based on the normal distribution assumptions of both $\mathbf{p}$ and $\mathbf{q}$ and the fact that these are normalised (see Sec. 3.1), we formulate the prior terms as the squared $L^{2}$ norms of the parameters:

$$
E_{\text {priors }}(\mathbf{p}, \mathbf{q})=c_{i d}\|\mathbf{p}\|^{2}+c_{\text {exp }}\|\mathbf{q}\|^{2}
$$

where $c_{i d}$ and $c_{\text {exp }}$ are constants that weight the contribution of the prior terms over identity and expression parameters respectively.

\subsubsection{Gauss-Newton Project-Out Optimisation}

Inspired by the extensive literature in Lucas-Kanade 2D image alignment [8], [24], [25], [26], [59], [60], we formulate a GaussNewton optimization framework to efficiently minimize the energy of Eq. (14).

Parameters update. The shape and camera parameters are updated in an additive manner, i.e.

$$
\mathbf{p} \leftarrow \mathbf{p}+\Delta \mathbf{p}, \quad \mathbf{q} \leftarrow \mathbf{q}+\Delta \mathbf{q}, \quad \mathbf{c} \leftarrow \mathbf{c}+\Delta \mathbf{c}
$$

where $\Delta \mathbf{p}, \Delta \mathbf{q}$ and $\Delta \mathbf{c}$ are their increments estimated at each fitting iteration. Note that in the case of the quaternion used to parameterize the 3D rotation matrix, the update is performed as the multiplication

$$
\begin{aligned}
\mathbf{q} & (\Delta \mathbf{q}) \mathbf{q}=\left[\begin{array}{c}
\Delta q_{0} \\
\Delta \mathbf{q}_{1: 3}
\end{array}\right]\left[\begin{array}{c}
q_{0} \\
\mathbf{q}_{1: 3}
\end{array}\right]= \\
& =\left[\begin{array}{c}
\Delta q_{0} q_{0}-\Delta \mathbf{q}_{1: 3}^{\top} \mathbf{q}_{1: 3} \\
\Delta q_{0} \mathbf{q}_{1: 3}+q_{0} \Delta \mathbf{q}_{1: 3}+\Delta \mathbf{q}_{1: 3} \times \mathbf{q}_{1: 3}
\end{array}\right]
\end{aligned}
$$

However, we will still denote it as an addition for simplicity. Finally, we found that it is beneficial to keep the focal length constant in most cases, due to its ambiguity with $t_{z}$. 
Linearisation. By introducing the additive incremental updates on the shape and camera parameters, the cost function is expressed as:

$$
\begin{aligned}
& E(\mathbf{p}+\Delta \mathbf{p}, \mathbf{q}+\Delta \mathbf{q}, \mathbf{c}+\Delta \mathbf{c}, \boldsymbol{\lambda})= \\
& \quad\|\mathbf{F}(\mathcal{W}(\mathbf{p}+\Delta \mathbf{p}, \mathbf{q}+\Delta \mathbf{q}, \mathbf{c}+\Delta \mathbf{c}))-\mathcal{T}(\boldsymbol{\lambda})\|^{2} \\
& \quad+c_{\ell}\left\|\mathcal{W}_{l}(\mathbf{p}+\Delta \mathbf{p}, \mathbf{q}+\Delta \mathbf{q}, \mathbf{c}+\Delta \mathbf{c})-\ell\right\|^{2} \\
& \quad+c_{i d}\|\mathbf{p}+\Delta \mathbf{p}\|^{2}+c_{e x p}\|\mathbf{q}+\Delta \mathbf{q}\|^{2}
\end{aligned}
$$

Note that the texture reconstruction and landmarks constraint terms of this cost function are non-linear due to the camera model operation. We need to linearise them around $(\mathbf{p}, \mathbf{q}, \mathbf{c})$ using first order Taylor series expansion at $(\mathbf{p}+\Delta \mathbf{p}, \mathbf{q}+\Delta \mathbf{q}, \mathbf{c}+\Delta \mathbf{c})=$ $(\mathbf{p}, \mathbf{q}, \mathbf{c}) \Rightarrow(\Delta \mathbf{p}, \Delta \mathbf{q}, \Delta \mathbf{c})=\mathbf{0}$. The linearisation for the image term gives:

$$
\begin{aligned}
& \mathbf{F}(\mathcal{W}(\mathbf{p}+\Delta \mathbf{p}, \mathbf{q}+\Delta \mathbf{q}, \mathbf{c}+\Delta \mathbf{c})) \approx \mathbf{F}(\mathcal{W}(\mathbf{p}, \mathbf{q}, \mathbf{c})) \\
& \quad+\mathbf{J}_{\mathbf{F}, \mathbf{p}} \Delta \mathbf{p}+\mathbf{J}_{\mathbf{F}, \mathbf{q}} \Delta \mathbf{q}+\mathbf{J}_{\mathbf{F}, \mathbf{c}} \Delta \mathbf{c}
\end{aligned}
$$

where:

$$
\mathbf{J}_{\mathbf{F}, \mathbf{p}}=\left.\nabla \mathbf{F} \frac{\partial \mathcal{W}}{\partial \mathbf{p}}\right|_{\mathbf{p}=\mathbf{p}}, \mathbf{J}_{\mathbf{F}, \mathbf{q}}=\left.\nabla \mathbf{F} \frac{\partial \mathcal{W}}{\partial \mathbf{q}}\right|_{\mathbf{q}=\mathbf{q}}, \mathbf{J}_{\mathbf{F}, \mathbf{c}}=\left.\nabla \mathbf{F} \frac{\partial \mathcal{W}}{\partial \mathbf{c}}\right|_{\mathbf{c}=\mathbf{c}}
$$

are the image Jacobians with respect to the identity, expression and camera parameters, respectively. Note that most dense featureextraction functions $\mathcal{F}(\cdot)$ are non-differentiable, thus we simply compute the gradient of the multi-channel feature image $\nabla \mathbf{F}$.

Similarly, the linearisation on the sparse landmarks projection term gives:

$$
\begin{aligned}
& \mathcal{W}_{l}(\mathbf{p}+\Delta \mathbf{p}, \mathbf{q}+\Delta \mathbf{q}, \mathbf{c}+\Delta \mathbf{c}) \approx \\
& \mathcal{W}_{l}(\mathbf{p}, \mathbf{q}, \mathbf{c})+\mathbf{J}_{L, \mathbf{p}} \Delta \mathbf{p}+\mathbf{J}_{L, \mathbf{q}} \Delta \mathbf{q}+\mathbf{J}_{L, \mathbf{c}} \Delta \mathbf{c}
\end{aligned}
$$

where: $\mathbf{J}_{L, \mathbf{p}}=\left.\frac{\partial \mathcal{W}_{l}}{\partial \mathbf{p}}\right|_{\mathbf{p}=\mathbf{p}}, \mathbf{J}_{L, \mathbf{q}}=\left.\frac{\partial \mathcal{W}_{l}}{\partial \mathbf{q}}\right|_{\mathbf{q}=\mathbf{q}}, \mathbf{J}_{L, \mathbf{c}}=\left.\frac{\partial \mathcal{W}_{l}}{\partial \mathbf{c}}\right|_{\mathbf{c}=\mathbf{c}}$ are the landmarks projection Jacobians. Please refer to the Supplementary Material for more details on the computation of these derivatives.

By substituting Eqs. (21) and (22) into Eq. (20) the cost function is approximated as:

$$
\begin{aligned}
& E(\mathbf{p}+\Delta \mathbf{p}, \mathbf{q}+\Delta \mathbf{q}, \mathbf{c}+\Delta \mathbf{c}, \boldsymbol{\lambda}) \approx \\
& \left\|\mathbf{F}(\mathcal{W}(\mathbf{p}, \mathbf{q}, \mathbf{c}))+\mathbf{J}_{\mathbf{F}, \mathbf{p}} \Delta \mathbf{p}+\mathbf{J}_{\mathbf{F}, \mathbf{q}} \Delta \mathbf{q}+\mathbf{J}_{\mathbf{F}, \mathbf{c}} \Delta \mathbf{c}-\mathcal{T}(\boldsymbol{\lambda})\right\|^{2} \\
& \quad+c_{\ell}\left\|\mathcal{W}_{l}(\mathbf{p}, \mathbf{q}, \mathbf{c})+\mathbf{J}_{L, \mathbf{p}} \Delta \mathbf{p}+\mathbf{J}_{L, \mathbf{q}} \Delta \mathbf{q}+\mathbf{J}_{L, \mathbf{c}} \Delta \mathbf{c}-\ell\right\|^{2} \\
& \quad+c_{i d}\|\mathbf{p}+\Delta \mathbf{p}\|^{2}+c_{\exp }\|\mathbf{q}+\Delta \mathbf{q}\|^{2}
\end{aligned}
$$

Adopting the Project-Out optimisation approach, we optimise on the orthogonal complement of the texture subspace which eliminates the need to consider a texture parameters increment at each iteration. In more detail, the minimisation of the energy of Eq. (23) with respect to $\boldsymbol{\lambda}$ can be expressed analytically as a function of the increments $\Delta \mathbf{p}, \Delta \mathbf{q}, \Delta \mathbf{c}$ :

$$
\begin{aligned}
\boldsymbol{\lambda}=\mathbf{U}_{t}^{\top}(\mathbf{F}(\mathcal{W}(\mathbf{p}, \mathbf{q}, \mathbf{c})) & +\mathbf{J}_{\mathbf{F}, \mathbf{p}} \Delta \mathbf{p} \\
& \left.+\mathbf{J}_{\mathbf{F}, \mathbf{q}} \Delta \mathbf{q}+\mathbf{J}_{\mathbf{F}, \mathbf{c}} \Delta \mathbf{c}-\overline{\mathbf{t}}\right)
\end{aligned}
$$

We plug this expression into Eq. (23) to eliminate the dependence of the energy on $\boldsymbol{\lambda}$ and we get the following minimisation problem:

$$
\begin{aligned}
& \underset{\Delta \mathbf{p}, \Delta \mathbf{q}, \Delta \mathbf{c}}{\arg \min } \\
& \left\|\mathbf{F}(\mathcal{W}(\mathbf{p}, \mathbf{q}, \mathbf{c}))+\mathbf{J}_{\mathbf{F}, \mathbf{p}} \Delta \mathbf{p}+\mathbf{J}_{\mathbf{F}, \mathbf{q}} \Delta \mathbf{q}+\mathbf{J}_{\mathbf{F}, \mathbf{c}} \Delta \mathbf{c}-\overline{\mathbf{t}}\right\|_{\mathbf{P}}^{2} \\
& +c_{\ell}\left\|\mathcal{W}_{l}(\mathbf{p}, \mathbf{q}, \mathbf{c})+\mathbf{J}_{L, \mathbf{p}} \Delta \mathbf{p}+\mathbf{J}_{L, \mathbf{q}} \Delta \mathbf{q}+\mathbf{J}_{L, \mathbf{c}} \Delta \mathbf{c}-\ell\right\|^{2} \\
& +c_{i d}\|\mathbf{p}+\Delta \mathbf{p}\|^{2}+c_{\exp }\|\mathbf{q}+\Delta \mathbf{q}\|^{2}
\end{aligned}
$$

where $\mathbf{P}=\mathbf{I}_{C N}-\mathbf{U}_{t} \mathbf{U}_{t}^{\top}$ is the orthogonal complement of the texture subspace that functions as the "project-out" operator. Note that in this formulation $\lambda$ plays no explicit role. Further note that in order to derive Eq. (25), we use the properties $\mathbf{P}^{\top}=\mathbf{P}$ and $\mathbf{P}^{\top} \mathbf{P}=\mathbf{P}$.

The problem of Eq. (25) is a linear least squares problem that can be written in the general compact form:

$$
\underset{\Delta \mathbf{b}}{\arg \min }\|\mathbf{J} \Delta \mathbf{b}-\mathbf{e}\|^{2}
$$

where $\Delta \mathbf{b}=\left[\Delta \mathbf{p}^{\top}, \Delta \mathbf{q}^{\top}, \Delta \mathbf{c}^{\top}\right]^{\top}$ is a vector with all the unknowns (incremental updates) and $\mathbf{J}$ is the overall Jacobian of the problem:

$$
\mathbf{J}=\left[\mathbf{J}_{\mathbf{p}}\left|\mathbf{J}_{\mathbf{q}}\right| \mathbf{J}_{\mathbf{c}}\right]=\left[\begin{array}{c|c|c}
\mathbf{P J}_{\mathbf{F}, \mathbf{p}} & \mathbf{P J}_{\mathbf{F}, \mathbf{q}} & \mathbf{P} \mathbf{J}_{\mathbf{F}, \mathbf{c}} \\
\sqrt{c_{\ell}} \mathbf{J}_{L, \mathbf{p}} & \sqrt{c_{\ell}} \mathbf{J}_{L, \mathbf{q}} & \sqrt{c_{\ell}} \mathbf{J}_{L, \mathbf{c}} \\
\sqrt{c_{i d}} \mathbf{I}_{n_{p}} & \mathbf{0}_{n_{p} \times n_{q}} & \mathbf{0}_{n_{p} \times n_{c}} \\
\mathbf{0}_{n_{q} \times n_{p}} & \sqrt{c_{e x p}} \mathbf{I}_{n_{q}} & \mathbf{0}_{n_{q} \times n_{c}}
\end{array}\right]
$$

where $\mathbf{0}_{m \times n}$ denotes the $m \times n$ zero matrix. Also, $\mathbf{e}$ is the overall offset vector of the problem:

$$
\mathbf{e}=\left[\begin{array}{c}
\mathbf{P}(\overline{\mathbf{t}}-\mathbf{F}(\mathcal{W}(\mathbf{p}, \mathbf{q}, \mathbf{c}))) \\
\sqrt{c_{\ell}}\left(\boldsymbol{\ell}-\mathcal{W}_{l}(\mathbf{p}, \mathbf{q}, \mathbf{c})\right) \\
-\sqrt{c_{i d}} \mathbf{p} \\
-\sqrt{c_{e x p}} \mathbf{q}
\end{array}\right]
$$

We compute $\Delta \mathrm{b}$ by solving the linear system that is derived from taking the gradient of the cost function in Eq. (26) and setting it to zero: $\left(\mathbf{J}^{\top} \mathbf{J}\right) \Delta \mathbf{b}=\mathbf{J}^{\top} \mathbf{e}$. This system is of a relatively small scale, therefore it is straightforward to implement its solution.

Note that the above-described Project-Out scheme is a very efficient approach to solving the Gauss-Newton iterations for minimising the cost function of Eq. (14). It has been shown that this is much faster than the more widely-used Simultaneous algorithm [23], [25], [61].

Residual masking. In practice, we apply a mask on the texture reconstruction residual of the Gauss-Newton optimisation, in order to speed-up the 3DMM fitting. This mask is constructed by first acquiring the set of visible vertices using z-buffering and then randomly selecting $K$ of them. By keeping the number of vertices small ( $K \approx 5000 \ll N$ ), we manage to greatly speed-up the fitting process without any accuracy penalty. This z-buffering and random sampling is performed per-iteration, allowing for changes in the self-occlusion state of vertices as the optimisation progresses.

\subsection{Fitting on Videos}

In the case of videos, we extend our energy minimisation formulation, described in the previous Sec. 4.1. Due to our separable identity and expression shape model, we can fix the identity parameters throughout the whole video, a significant constraint 
that greatly helps our estimations. In addition, we impose temporal smoothness on the expression parameters, which improves the estimation of the 3D facial deformations of the individual observed in the input video. Furthermore, we can get a fast and accurate initialisation for the minimisation of the proposed energy by employing Structure from Motion on the per-frame sparse 2D landmarks with an efficient linear least squares fitting approach.

\subsubsection{Proposed Energy Formulation}

Let us assume that the input video consists of $n_{f}$ images, $\mathbf{I}_{1}, \ldots, \mathbf{I}_{f}, \ldots, \mathbf{I}_{n_{f}}$. As in the single-image case, we are based on the feature-based representation $\mathbf{F}_{f}=\mathcal{F}\left(\mathbf{I}_{f}\right)$ of the image of every frame $f=1, \ldots, n_{f}$. Also, let $\ell_{f}=$ $\left[x_{1 f}, y_{1 f}, \ldots, x_{L f}, y_{L f}\right]^{\top}$ be the $2 \mathrm{D}$ facial landmarks for the $f$-th frame. We are still denoting by $\mathbf{p}$ the identity parameters vector, which as already mentioned, is fixed over all frames of the video. However, we consider that every frame has its own expression, camera, and texture parameters vectors, which we denote by $\mathbf{q}_{f}, \mathbf{c}_{f}$ and $\boldsymbol{\lambda}_{f}$ respectively. We also denote by $\hat{\mathbf{q}}, \hat{\mathbf{c}}$ and $\hat{\lambda}$ the concatenation of the corresponding parameter vectors over all frames: $\hat{\mathbf{q}}^{\top}=\left[\mathbf{q}_{1}^{\top}, \ldots, \mathbf{q}_{n_{f}}^{\top}\right], \hat{\mathbf{c}}^{\top}=\left[\mathbf{c}_{1}^{\top}, \ldots, \mathbf{c}_{n_{f}}^{\top}\right]$ and $\hat{\boldsymbol{\lambda}}^{\top}=\left[\boldsymbol{\lambda}_{1}^{\top}, \ldots, \boldsymbol{\lambda}_{n_{f}}^{\top}\right]$.

To fit the 3DMM on a video, we propose to minimise the following energy, which is a multi-frame extension of the energy in Eq. (14):

$$
\begin{aligned}
\hat{E}(\mathbf{p}, \hat{\mathbf{q}}, \hat{\mathbf{c}}, \hat{\boldsymbol{\lambda}}) & =\hat{E}_{\text {text }}(\mathbf{p}, \hat{\mathbf{q}}, \hat{\mathbf{c}}, \hat{\boldsymbol{\lambda}})+c_{\ell} \hat{E}_{\text {land }}(\mathbf{p}, \hat{\mathbf{q}}, \hat{\mathbf{c}}) \\
& +\hat{E}_{\text {priors }}(\mathbf{p}, \hat{\mathbf{q}})+c_{s m} \hat{E}_{\text {smooth }}(\hat{\mathbf{q}})
\end{aligned}
$$

where $\hat{E}_{\text {text }}, \hat{E}_{\text {land }}$ and $\hat{E}_{\text {priors }}$ are the multi-frame extensions of the texture reconstruction, 2D landmarks term and prior regularisation terms respectively. Furthermore, $\hat{E}_{\text {smooth }}$ is a temporal smoothness term that we impose on the time-varying expression parameters $\mathbf{q}_{f}$. Also $c_{\ell}$ and $c_{s m}$ are the balancing weights for the terms $\hat{E}_{\text {land }}$ and $\hat{E}_{\text {smooth }}$ respectively. Next, we present every term of the energy in more detail.

The texture reconstruction term $\left(\hat{E}_{\text {text }}\right)$ is the main data term and sums the texture reconstruction error from all frames:

$$
\hat{E}_{\text {text }}(\mathbf{p}, \hat{\mathbf{q}}, \hat{\mathbf{c}}, \hat{\boldsymbol{\lambda}})=\sum_{f=1}^{n_{f}}\left\|\mathbf{F}_{f}\left(\mathcal{W}\left(\mathbf{p}, \mathbf{q}_{f}, \mathbf{c}_{f}\right)\right)-\mathcal{T}\left(\boldsymbol{\lambda}_{f}\right)\right\|^{2}
$$

The 2D landmarks term ( $\left.\hat{E}_{\text {land }}\right)$ is a summation of the reprojection error of the sparse 2D landmarks for all frames:

$$
\hat{E}_{\text {land }}(\mathbf{p}, \hat{\mathbf{q}}, \hat{\mathbf{c}})=\sum_{f=1}^{n_{f}}\left\|\mathcal{W}_{l}\left(\mathbf{p}, \mathbf{q}_{f}, \mathbf{c}_{f}\right)-\boldsymbol{\ell}_{f}\right\|^{2}
$$

The shape priors term ( $\left.\hat{E}_{\text {priors }}\right)$ imposes priors on the reconstructed 3D facial shape of every frame. Since the facial shape at every frame is derived from the (zero-mean and unit-variance) identity parameter vector $\mathbf{p}$ and the frame-specific expression parameter vector $\mathbf{q}_{f}$ (also zero-mean and unit-variance), we define this term as:

$$
\begin{aligned}
\hat{E}_{\text {priors }}(\mathbf{p}, \hat{\mathbf{q}}) & =\hat{c}_{i d}\|\mathbf{p}\|^{2}+c_{\text {exp }} \sum_{f=1}^{n_{f}}\left\|\mathbf{q}_{f}\right\|^{2} \\
& =\hat{c}_{i d}\|\mathbf{p}\|^{2}+c_{\text {exp }}\|\hat{\mathbf{q}}\|^{2}
\end{aligned}
$$

where $\hat{c}_{i d}$ and $c_{\text {exp }}$ are the balancing weights for the prior terms of identity and expression respectively.
The temporal smoothness term ( $\left.\hat{E}_{\text {smooth }}\right)$ is video-specific and enforces smoothness on the expression parameters vector $\mathbf{q}_{f}$ by penalising the squared norm of the discrimination of its $2^{\text {nd }}$ temporal derivative. This corresponds to the regularisation imposed in smoothing splines and leads to naturally smooth trajectories over time. More specifically, this term is defined as:

$$
\hat{E}_{\text {smooth }}(\hat{\mathbf{q}})=\sum_{f=2}^{n_{f}-1}\left\|\mathbf{q}_{f-1}-2 \mathbf{q}_{f}+\mathbf{q}_{f+1}\right\|^{2}=\left\|\mathbf{D}^{2} \hat{\mathbf{q}}\right\|^{2}
$$

where the summation is done over all frames for which the discretised $2^{\text {nd }}$ derivative can be expressed without having to assume any form of padding outside the temporal window of the video. Also $\mathbf{D}^{2}: \mathbb{R}^{n_{q} n_{f}} \rightarrow \mathbb{R}^{n_{q}\left(n_{f}-2\right)}$ is the linear operator that instantiates the discretised $2^{\text {nd }}$ derivative of the $n_{q}$-dimensional vector $\mathbf{q}_{f}$. This means that $\mathbf{D}^{2} \hat{\mathbf{q}}$ is a vector that stacks the vectors $\left(\mathbf{q}_{f-1}-2 \mathbf{q}_{f}+\mathbf{q}_{f+1}\right)$, for $f=2, \ldots, n_{f}-1$. It is worth mentioning that we could have imposed temporal smoothness on the parameters $\mathbf{c}_{f}, \boldsymbol{\lambda}_{f}$ too. However, we have empirically observed that the temporal smoothness on $\mathbf{q}_{f}$, in conjunction with fixing the identity parameters $\mathbf{p}$ over time, is adequate for accurate and temporally smooth estimations.

\subsubsection{Initialisation}

The proposed energy $\hat{E}$ in Eq. (29) is highly non-convex, therefore a good initialisation is of paramount importance. To achieve highly-accurate fitting results on videos, even in especially challenging cases, we design a computationally efficient video initialisation strategy, by decomposing the problem into two simpler ones that can be solved quickly and accurately.

For the above reasons, we consider for this part a scaled orthographic camera, which simplifies the optimisation by making the projection function $\pi\left(\mathbf{c}_{\text {intr }}, \mathbf{v}\right)$ described in Eq. (6) to be linear with respect to $\mathbf{v}$. Also, we are based on a simplified version of the proposed energy $\hat{E}$ in Eq. (29) that does not contain the texture reconstruction term:

$$
\begin{aligned}
\hat{E}_{\text {init }}(\mathbf{p}, \hat{\mathbf{q}}, \hat{\mathbf{c}}) & =c_{\ell} \hat{E}_{\text {land }}(\mathbf{p}, \hat{\mathbf{q}}, \hat{\mathbf{c}}) \\
& +\hat{E}_{\text {priors }}(\mathbf{p}, \hat{\mathbf{q}})+c_{s m} \hat{E}_{\text {smooth }}(\hat{\mathbf{q}})
\end{aligned}
$$

This means that the only data term is $\hat{E}_{\text {land }}$ and the estimations use only the sparse 2D landmarks as input. Full details are provided in the Supplementary Material.

\subsubsection{Video-Specific Texture Model}

Apart from offering a good starting point for the main optimisation, the initialisation described in the previous sections is first of all used to bootstrap the learning of the video-specific texture model, as described in Sec. 3.3. To improve the computational efficiency of this procedure, we down-sample the frames and only consider 1 every $f_{\text {step }}$ frames. In more detail, using the estimated shape and camera parameters of the considered frames, we sample the facial texture $\mathbf{t}_{f}=\mathbf{F}_{f}\left(\mathcal{W}\left(\mathbf{p}, \mathbf{q}_{\mathbf{f}}, \mathbf{c}_{\mathbf{f}}\right)\right)$ and utilise it in the Principal Component Pursuit (PCP) problem of Eq. (12).

\subsubsection{Main Optimisation of the Proposed Energy}

Similarly to the single-image case (Sec. 4.1.2), we minimise the proposed energy $\hat{E}$ of Eq. (29) by following a Gauss-Newton scheme. In every iteration, we consider the current estimates $\mathbf{p}, \hat{\mathbf{q}}$, $\hat{\mathbf{c}}$ and we linearise the texture reconstruction and landmarks error functions around them. After this approximation, the problem 
becomes a linear least squares problem with respect to the texture parameters $\hat{\lambda}$ and the incremental updates $\Delta \mathbf{p}, \Delta \hat{\mathbf{q}}$ and $\Delta \hat{\mathbf{c}}$. For more details, please see Supplementary Material.

Regarding the unknown texture parameters, we follow again the Project-Out approach. In more detail, the minimisation with respect to each $\boldsymbol{\lambda}_{f}$ is decoupled in every frame and can be found analytically as a function of $\Delta \mathbf{p}, \Delta \hat{\mathbf{q}}_{f}$ and $\Delta \hat{\mathbf{c}}_{f}$, exactly as in Eq. (24) (see Supplementary Material). Using this expression in the expression of the linearised energy $\hat{E}$, we derive the following problem:

$$
\underset{\Delta \mathbf{p}, \Delta \hat{\mathbf{q}}, \Delta \hat{\mathbf{c}}}{\arg \min } \hat{E}(\mathbf{p}+\Delta \mathbf{p}, \hat{\mathbf{q}}+\Delta \hat{\mathbf{q}}, \hat{\mathbf{c}}+\Delta \hat{\mathbf{c}})
$$

The above problem is a large-scale linear least squares problem that can be written in the form (see Supplementary Material for detailed derivations):

$$
\underset{\Delta \hat{\mathbf{b}}}{\arg \min }\|\hat{\mathbf{J}} \Delta \hat{\mathbf{b}}-\hat{\mathbf{e}}\|^{2}
$$

where $\Delta \hat{\mathbf{b}}=\left[\Delta \mathbf{p}^{\top}, \Delta \hat{\mathbf{q}}^{\top}, \Delta \hat{\mathbf{c}}^{\top}\right]$ is a vector with all the unknown incremental updates from all the frames. Also, $\hat{\mathbf{J}}$ is the corresponding overall Jacobian matrix that has a sparse structure. Finally, $\hat{\mathbf{e}}$ is the overall error term. Note that the dimensionality of $\Delta \hat{\mathbf{b}}$ (and hence the number of parameters to estimate) is $N_{\text {tot }}=n_{p}+n_{f}\left(n_{q}+7\right)$ and the Jacobian $\hat{\mathbf{J}}$ is of size $\left(n_{f}\left(C N+2 L+n_{q}+1\right)-2 n_{q}\right) \times N_{\text {tot }}$. Given the fact that we consider hundreds of frames $n_{f}$ and tens of thousands of vertices $N$, the least square problem (36) is a very large-scale one. For example, for the choice of parameters considered in our experiments, the Jacobian $\hat{\mathbf{J}}$ is of size $425,884,944 \times 35,100$. This is in contrast to the corresponding problem of the single-image fitting case, where the problem was of small scale, so we could solve it by standard approaches. Therefore, we follow a videospecific strategy, in order to achieve a satisfactory scalability. In more detail, we consider the equivalent linear system (derived by equating the gradient to zero): $\hat{\mathbf{J}}^{\top} \hat{\mathbf{J}} \mathbf{x}=\hat{\mathbf{J}}^{\top} \mathbf{b}$ and adopt an efficient and parallelisable method that avoids the explicit computation and storage of the matrices $\hat{\mathbf{J}}$ and $\left(\hat{\mathbf{J}}^{\top} \hat{\mathbf{J}}\right)$, which are very large-scale and sparse. More precisely, following other recent methods of 3D facial and more general deformable surface reconstruction [48], [62], we use a preconditioned conjugate gradient (PCG) solver, for which we only need to efficiently implement functions that compute the multiplications $\left(\hat{\mathbf{J}}^{\top} \hat{\mathbf{J}}\right) \mathbf{x}$ and $\hat{\mathbf{J}}^{\top} \mathbf{h}$ for any input vectors $\mathbf{x}$ and $\mathbf{h}$. For the preconditioning, we use the inverses of the diagonal blocks of $\hat{\mathbf{J}}^{\top} \hat{\mathbf{J}}$.

\section{BenchmaRKS FOR 3DMM ImAGe AND VideO FITTING}

To allow for the quantitative evaluation of our proposed 3DMM image and video fitting methods, we have constructed three benchmarks - KF-ITW, 3dMDLab and 4DMaja. For the benefit of the research community, we are making these benchmarks publicly available.

The error metric we use in all 3D shape recovery benchmarks is as follows. All raw ground truth meshes are brought into correspondence with a reference template by employing Nonrigid ICP guided by manual annotations. We use a slightly modified variant of the mean of the Basel Face Model [17] (BFM) as a standard here (we just remove the inner-mouth and nostril parts). Each model under test is also corresponded with this
BFM template using the same annotation-driven Nonrigid ICP deformation. We now can densely relate a 3D fit from any model with the ground truth mesh via the correspondence mappings, and we use this to define a per-vertex error metric, specifically the per-vertex Euclidean error between the recovered shape and the model-specific corresponded ground-truth fit, normalized by the inter-ocular distance for the test mesh (i.e. the distance between the outer corners of the eyes). Only regions of the face that are recovered by all methods under test are evaluated, so no model is penalized for not having complete coverage. In practice for these experiments, this corresponds to the inner region of the face (i.e. inside the jawline). See Fig. 4 in the Supplementary Material for an example of the region of evaluation.

\subsection{KF-ITW Dataset}

The first dataset we introduce is focused on providing quantitative evaluation for 3DMM image fitting. KF-ITW is, to the best of our knowledge, the first dataset where ground truth 3D facial shape is provided along with images captured under relatively unconstrained conditions.

The dataset consists of 17 different subjects captured under various illumination conditions performing a range of expressions (neutral, happy, surprise). We employed the KinectFusion [63], [64] framework to acquire a 3D representation of the subjects with a Kinect v1 sensor. In order to accurately reconstruct the entire surface of the face, each subject was instructed to stay still in a fixed pose whilst a circular motion scanning pattern was carried out around the face. The fused mesh for each subject recovered from KinectFusion serves as a 3D face ground-truth in which we can evaluate our algorithm and compare it to other methods. Single frames picked from the RGB video stream of the Kinect sensor recording are selected as input images of our benchmark. The frame rate for every subject was constant to 8 frames per second, and a voxel grid of size $608^{3}$ was utilised to get the detailed 3D scans of the faces. After getting the 3D scans from the KinectFusion framework we manually annotate each mesh with the iBUG 49 sparse landmark set, and correspond all meshes with the BFM reference mesh as described at the start of this section.

Although a short video sequence is captured as part of the acquisition process, we do not consider KF-ITW a suitable dataset for video facial shape recovery evaluation, as this video is highly contrived (the user is requested to hold still the entire time and the video motion is very specific and unnatural). To this end we only supply single frames from the acquisition process for image-fitting evaluation.

We use the ground-truth manual annotations of sparse landmarks provided in the KF-ITW dataset to initialize and fit each technique under test to the "in-the-wild" style images in the dataset.

\subsection{3dMDLab Benchmark}

To quantitatively evaluate additional aspects of 3DMM image fitting, we are introducing a second benchmark, which we call "3dMDLab". In contrast to KF-ITW, this benchmark has been created in controlled conditions using a high-resolution $3 d M D^{T M}$ facial scanner. This results in highly-detailed ground truth facial meshes and is therefore suited to evaluating 3D fitting methods on constrained rather than "in-the-wild" data. In more detail, 3dMDLab includes 4 subjects each performing 2 different expressions for a total of eight 3D face scans. It includes 8 real images 


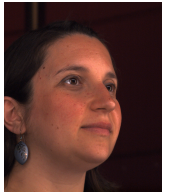

(a)

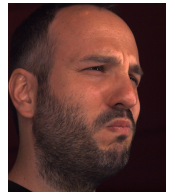

(b)

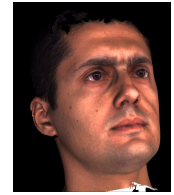

(c)

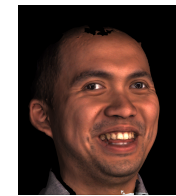

(d)
Fig. 4. 3dMDLab benchmark: (a,b) Examples of 2 out of 8 images of 3dMDLab-real set. We introduce this benchmark to evaluate image fitting methods under ideal conditions. (c,d) Examples of 2 out of 8 images of 3dMDLab-synthetic set. We introduce this benchmark to evaluate image fitting methods under synthetic strong illumination conditions.
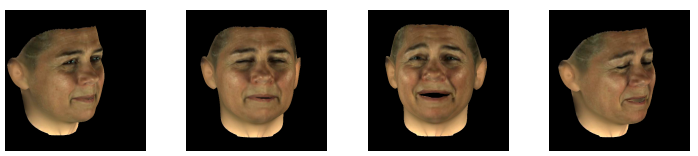

Fig. 5. 4DMaja-synthetic benchmark video: 4 out of 440 frames of a synthetic video created using high-resolution 4D face scans and rendering using a synthetic camera under varying 3D pose. Since this is a rendered video, it is accompanied by $4 \mathrm{D}$ ground truth mesh information.

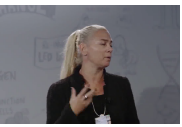

(a)

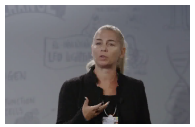

(b)

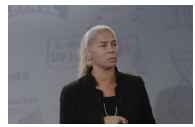

(c)

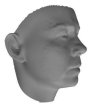

(d)
Fig. 6. 4DMaja-real benchmark video: (a-c) 3 out of 387 frames of a real video under "in-the-wild" conditions. (d) Ground truth mesh representing the shape identity component of the 3D facial shape of the captured subject.

(“3dMDLab-real”) in ideal, laboratory conditions, coming directly from one of the RGB cameras of the $3 d M D^{T M}$ face scanning system, see e.g. Fig. 4(a,b). It also includes 8 synthetic images ("3dMDLab-synthetic") created by the same scans after rendering them from different view points with varying synthetic light, see e.g. Fig. 4(c,d). All real and synthetic images are high-resolution $(2048 \times 2448$ pixels $)$ images with true colour range $(24$ bits per pixel). See the Supplementary Material for further details.

\subsection{DMaja Benchmark}

To quantitatively evaluate 3DMM video fitting, we are introducing a third benchmark, which we call "4DMaja". To the best of our knowledge, this is the first publicly available benchmark that allows detailed quantitative evaluation of $3 \mathrm{D}$ face reconstruction on videos. 4DMaja includes two videos of the same subject (Prof. Maja Pantic) exhibiting various natural expressions and head pose variations. The first video ("4DMaja-real") is a 440 frame synthetic sequence generated from high-resolution face scans taken using a $D I 4 D^{T M}$ face scanner, with the (virtual) camera undergoing a periodic rotation (Fig. 5). This allows for a quantitative evaluation of the 3D face reconstruction for every frame of the video.

The second video ("4DMaja-synthetic") is a 387 frame clip from a public talk the subject gave and hence is under "in-thewild" conditions. We associate with this video a high-resolution neutral 3D scan of the subject which was taken with a $D I 4 D^{T M}$ scanner within 2 months of the talk (Fig. 6). Given the small time difference we can consider the 3D scan a ground truth of the identity component of the 3D facial shape for the real video. In this way we can quantitatively evaluate how well the 3D facial identity is estimated when different methods are run over a real "in-the-wild" video for the first time.

\section{EXPERIMENTS}

In this section we present in-depth qualitative and quantitative evaluations of our proposed image and video fitting methods. Apart from comparisons with classic and state-of-the-art methods, we are presenting self-evaluations of our fitting framework by comparing results obtained under different settings. We label our "in-the-wild" image and video fitting methods as ITW and ITW$V$ respectively. Further details, visualisations and experiments are presented in the Supplementary Material.

We use two different variants of our adopted 3DMM model of shape and texture variation, obtained by using either the Basel Face Model (BFM) [17] or the LSFM model [18] as 3D shape models for identity variation. This is denoted by the labels "(Basel)" and "(LSFM)" after the names of our methods, for example "ITW(Basel)" or "ITW-V(LSFM)". Note that while LSFM is a more accurate and powerful model, we are also adopting BFM in the experiments for the sake of fairness towards the methods that we compare with, which use BFM or other models of much smaller scale than LSFM. We expand the adopted models for identity variation by incorporating a model for expression variation provided by [16], following the process described in Sec. 3.1. We trained our "in-the-wild" texture model on the images of iBUG, LFPW \& AFW datasets [15] as described in Sec. 3.3 using the 3D shape fits provided by [65].

In the case of our image fitting method, the sparse 2D landmarks that are required as part of the input are either manually annotated (KF-ITW benchmark and input images of Fig. 7) or recovered using the CNN-based landmarker of [66] (3dMDLab benchmark). In the case of our video fitting method, the sequence of sparse 2D landmarks is extracted by either using a state-of-theart facial tracker from [67] (300VW videos - Fig. 11) or applying the CNN-based landmarker of [66] on every frame independently (4DMaja benchmark).

In the subsequent experimental evaluation, comparisons are performed with several existing methods for 3DMM fitting:

- "Classic": this is an implementation of the classic 3DMM fitting [44] with the original Basel laboratory texture model and full lighting equation. In all conducted experiments, we fed this method with the same sparse landmarks as our method.

- "Linear": this is the linear shape model fitting proposed in [20], [68]. For this method we use the Surrey Model with related blendshapes along with the implementation given in [68]. The methodology requires no texture model and fits to sparse landmarks. In all conducted experiments, we fed this method with the same sparse landmarks as our method. For the experiments on 3DMM fitting on videos, we applied this method on every frame independently and resulted to a procedure that was similar to the fitting performed by 4 DFace ${ }^{2}$ code.

- "3DMMedges": this is the 3DMM fitting method that was recently proposed by Bas et al. [69]. This method is fully automatic and uses landmarks and edge features. For this method, we used the publicly available source code ${ }^{3}$, following the provided demo

\footnotetext{
2. http://www.4dface.org/

3. https://github.com/waps101/3DMM_edges
} 


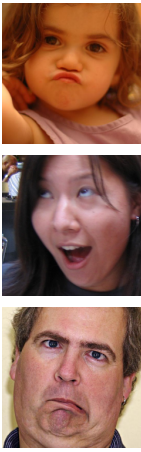

Input
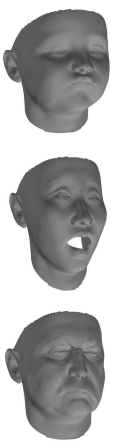

Ours
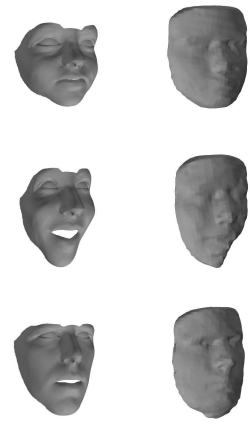

(a)

(b)
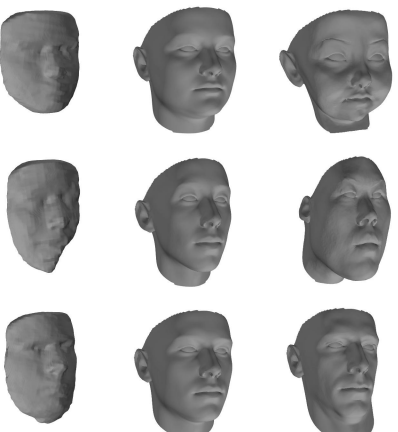

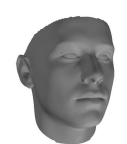

(c)

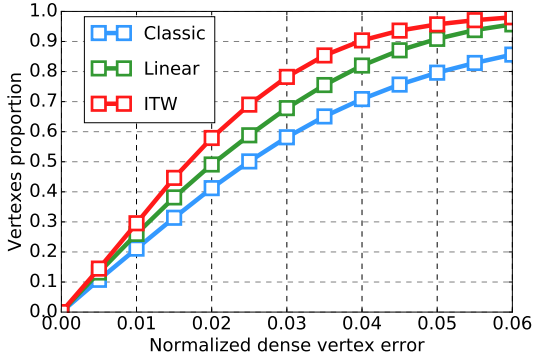

Fig. 7. 3D face reconstruction of challenging face images: qualitative comparison of our method (ITW(LSFM)) with other methods: (a) MoFA, (b) Jackson et al. 2017, (c) 3DMMedges (d) Classic.

function with its default parameters and without making any modification.

- "Jackson et al. 2017": this is a very recent method proposed Jackson et al. [70]. It performs 3D face reconstruction from a single image based on Convolutional Neural Networks [70]. It has been reported to achieve promising performance in unconstrained scenarios. To obtain results from this method, we have used the online demo provided by the authors ${ }^{4}$, where we only had to provide the input image.

- "MoFA": this is another very recent method, which was proposed by Tewari et al. [71]. It adopts a model-based deep convolutional autoencoder to perform 3D face reconstruction from a single "inthe-wild" image. Results of this method for a set of "in-the-wild" images were provided to us by the authors of this method.

Please note that Classic and 3DMMedges are using a 3D face model for identity variation, without incorporating any model for expression variation. Therefore, they are not reconstructing 3D shape deformations due to expressions and it is natural to yield less accurate results on emotive faces. All other tested methods are using a model of both identity and expression variation.

\subsection{DMM fitting on single images}

We present both qualitative and quantitative results and comparisons of our proposed "in-the-wild" model on single images.

Fig. 1 demonstrates qualitative results of our image fitting method on a wide range of fits of "in-the-wild" images drawn from the Helen and 300W datasets [14], [15] that qualitatively highlight the effectiveness of the proposed technique. To obtain these results, the BFM model has been used as the identity component of the shape model. We note that in a wide variety of expression, identity, lighting and occlusion conditions our model is able to robustly reconstruct a realistic $3 \mathrm{D}$ facial shape that stands up to scrutiny.

Fig. 7 shows qualitative comparisons of our ITW method (using LSFM shape identity model) with four existing techniques (MoFA, Jackson et al. 2017, 3DMMedges and Classic) on challenging images of faces exhibiting strong expressions. Subjectively, we observe that the results of our method are the most visually appealing. In contrast to all other tested methods, our method yields 3D face reconstructions that recover both the anatomical characteristics and the facial expressions of the

4. http://cvl-demos.cs.nott.ac.uk/vrn/
Fig. 8. Accuracy results for facial shape estimation on the KF-ITW database. The results are presented as CEDs of the normalized dense vertex error. Table 1 reports additional measures.

\begin{tabular}{|l|lc|}
\hline Method & AUC & Failure Rate (\%) \\
\hline \hline ITW & $\mathbf{0 . 6 7 8}$ & $\mathbf{1 . 7 9}$ \\
Linear & 0.615 & 4.02 \\
Classic & 0.531 & 13.9 \\
\hline \multicolumn{3}{|c|}{ TABLE 1 }
\end{tabular}

Accuracy results for facial shape estimation on the KF-ITW database. The table reports the Area Under the Curve (AUC) and Failure Rate of the CEDs of Fig. 8.

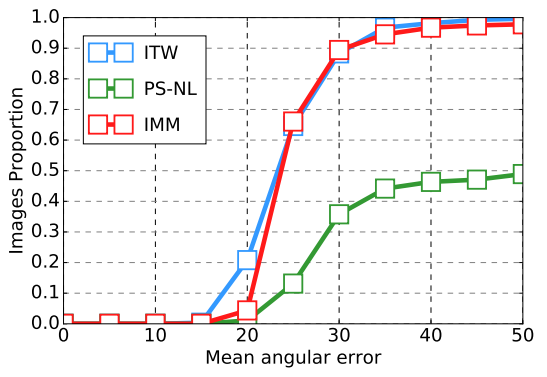

Fig. 9. Accuracy results for facial surface normal estimation on 100 subjects from the Photoface database [72]. The results are presented as CEDs of mean angular error.

captured subjects in a plausible way. We believe such results are unprecedented for such challenging conditions.

We also perform a quantitative evaluation on the KF-ITW benchmark, comparing our ITW(Basel) method with Linear and Classic techniques. Fig. 8 shows the Cumulative Error Distribution (CED) for this experiment for the three methods under comparison. Table 1 reports the corresponding Area Under the Curve (AUC) and failure rates. The Classic model struggles to fit to the "in-the-wild" conditions present in the test set, and performs the worst. The texture-free Linear model does better, but our ITW(Basel) model is most able to recover the facial shapes possibly due to its ideal feature basis for the "in-the-wild" conditions.

As a second quantitative evaluation, we employ images of 100 subjects from the Photoface database [72]. We use our ITW(Basel) method to find per-pixel normals and compare against two well established Shape-from-Shading (SfS) techniques: $P S-N L$ [73] and $I M M$ [42]. As a set of four illumination conditions are provided for each subject then we can generate ground-truth facial surface normals using calibrated 4-source Photometric Stereo [74]. In Fig. 9 we show the CED in terms of the mean angular error. $I T W$ slightly outperforms IMM even though both IMM and PS-NL use all four available images of each subject.

Apart from "in-the-wild" conditions like in the previous experiments, we evaluate and compare our fitting method under ideal, 


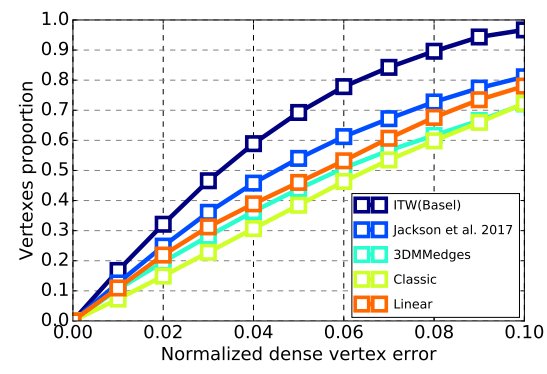

Fig. 10. Facial shape estimation on 3dMDLab-real: quantitative comparison of our image fitting method ITW(Basel) with other methods. The results are presented as CEDs of the normalised dense vertex error.

laboratory conditions. For this, we use the 8 images of 3dMDLabreal and compare our ITW(Basel) method with Linear, Classic, 3DMMedges and Jackson et al. 2017. Fig. 10 shows the CED for this experiment. We observe that our method yields a significantly better performance than the compared methods. This suggests that even under more controlled conditions, our image fitting approach is still advantageous over previous approaches.

\subsection{DMM fitting on videos}

In addition to the $3 \mathrm{D}$ shape recovery of single images we are also evaluating the available techniques on the task of $3 \mathrm{D}$ face reconstruction in the two videos of 4DMaja benchmark as well as in "in-the-wild" videos collected from the 300VW [75] dataset.

In our first experiment, we run ITW-V on 4DMaja-synthetic video (which provides a ground truth mesh for each frame of the sequence), and compare against "3DMMedges" [69], "Classic" [43], [44] and "Linear" [20], [68]. For each examined technique, we calculated an error at each frame of the sequence by computing the average per-vertex error between the recovered mesh and the corresponding ground truth. Fig. 12(a) shows that ITW-V outperforms "3DMMedges", which is the second best algorithm, by a large margin. Fig. 12(b) further shows how the per-frame error changes over time. Here, the significantly lower temporal error variance of ITW-V vindicates our decision to regularise identity and enforce smooth expressions over video sequences.

In the next evaluation scenario we run ITW-V on the "in-thewild" 4DMaja-real video (which, as a reminder, provides a single ground truth neutral expression mesh). In this case the error is based on comparing the mean recovered mesh for each method across the whole sequence with the single ground truth. In Fig. 13 it can be seen that ITW-V recovers identity more effectively than any other method.

The capability of ITW-V to reconstruct the 3D facial shape in "in-the-wild" videos is further examined by applying it to videos of the $300 \mathrm{VW}$ [75] dataset. For comparison, we both fit our ITW model to each frame individually with no video-specific cost (ITW per-frame) and show our full ITW video cost pipeline (ITW-V). Fig. 11 shows the representative frames from fitting the videos. We observe that in general both our ITW techniques visually outperform the SfM, Classic and Linear techniques in these challenging videos. We note that ITW-V, our video-specific fitting technique, combines the stability of Structure from Motion (SfM) with the detail from the ITW per-frame fitting. The Classic technique's explicit lighting model struggles to model "in-thewild" effects such as the microphone occlusion (first frame, first video) leading to the algorithm diverging. We note further that

\begin{tabular}{|l|cc|}
\hline Method & AUC & Failure Rate (\%) \\
\hline \hline ITW & $\mathbf{0 . 6 3 2}$ & $\mathbf{4 . 3 0}$ \\
RGB-MM & 0.610 & 6.13 \\
Classic & 0.545 & 10.9 \\
\hline \multicolumn{3}{|c|}{ TABLE 2 }
\end{tabular}

Facial shape estimation on 3dMDLab-synthetic: comparison of ITW (our fitting method), RGB-MM (a simplified version of our method where we have replaced the ITW texture model with an RGB texture model) and Classic 3DMM fitting [43], showing Area Under the Curve (AUC) and Failure Rate of the CED for each method.

\begin{tabular}{|l|lc|}
\hline Method & AUC & Failure Rate (\%) \\
\hline \hline ITW-V & $\mathbf{0 . 7 9 3}$ & $\mathbf{2 . 3 3}$ \\
ITW-V, init & 0.770 & 2.46 \\
\hline
\end{tabular}

3D identity shape estimation on 4DMaja-real video: self-evaluation of our fitting framework, comparing our video fitting method (ITW-V) with the initialisation of our video fitting method from sparse landmarks as described in Sec. 4.2.2 (ITW-V, init). Reported as Area Under the Curve (AUC) and Failure Rate of the CED for each method.

ITW-V does not suffer from drift in the identity of the individual (as ITW per-frame does, first video) or non-smooth expression changes (see ITW per-frame, second video in Supplementary Material). Finally, we also show in the bottom of this figure how our technique behaves in "in-the-wild" videos when used with the LSFM shape model. We have found this combination of ITW$\mathrm{V}$ with LSFM to be particularly effective, with LSFM providing excellent robustness to variations in age, gender, and ethnicity.

A video showing 3D reconstructions from the different methods tested is available on this paper's website ${ }^{5}$.

\subsection{Self-Evaluation of the proposed method}

To decouple the effect on performance of the texture model and the optimisation strategy employed, we present a self-evaluation of our fitting method, where we compare the following:

(i) a full version of our image fitting method (ITW), using the shape variation from BFM [17],

(ii) a version of our image fitting method where we have replaced the learned ITW texture model with the an RGB texture model (laboratory conditions), as provided by BFM [17]. We call this simplified version of our method "RGB-V".

(iii) an implementation of the classic 3DMM fitting ('Classic') [46], which uses the same texture and shape model as in (ii), coming from BFM [17].

This comparison sheds light on the benefits of using an ITW texture model and the proposed energy formulation independently. Table 2 presents the quantitative results of the above three methods on 3dMDLab-synthetic. We observe that method (ii) outperforms method (iii), which suggests that the proposed energy formulation is indeed beneficial as compared to the standard formulation followed by the classic 3DMM fitting. In addition, we observe that method (i) outperforms method (ii), which suggests that the proposed ITW texture is indeed beneficial as compared to the conventional RGB texture model. A second direction of selfevaluation is to compare our proposed video fitting method ITW$\mathrm{V}$ (Basel) against our image fitting, when the latter is applied to the frames of a video independently (ITW(Basel), per-frame). We employed this experimental setting on the 4DMaja-synthetic and calculated a mean error at each frame by averaging the differences

5. https://ibug.doc.ic.ac.uk/resources/itwmm 


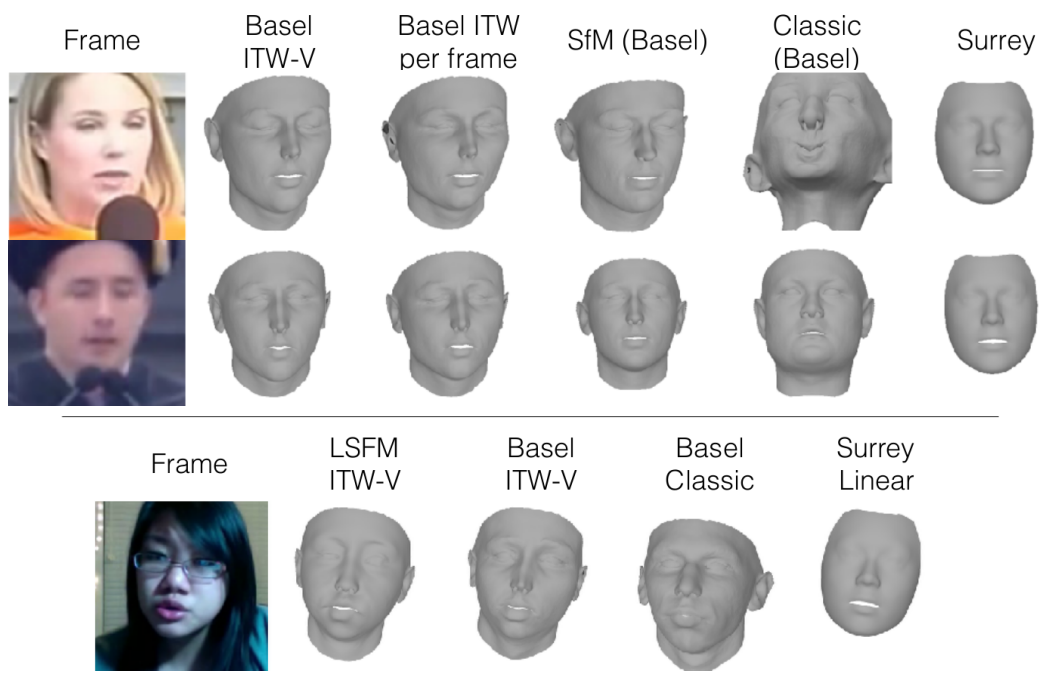

Fig. 11. 3D face reconstruction of videos from 300VW dataset [75]. Top: Two sample frames extracted from "in-the-wild" videos along with the 3D reconstructions performed using a variety of techniques. Bottom: A final qualitative comparison demonstrating how our proposed technique works well with a range of shape models, including the diverse Large Scale Facial Model (LSFM).

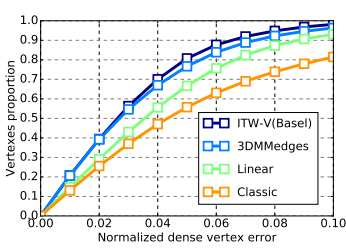

(a)

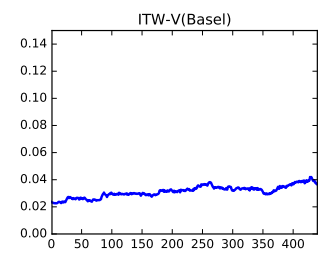
400

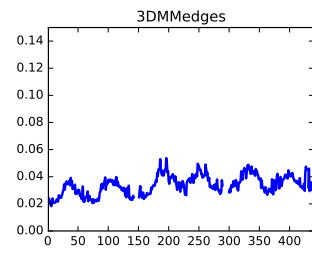

(b)

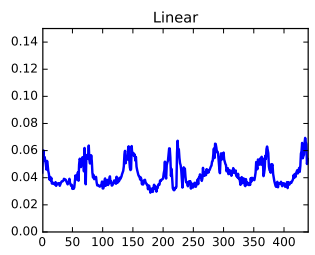

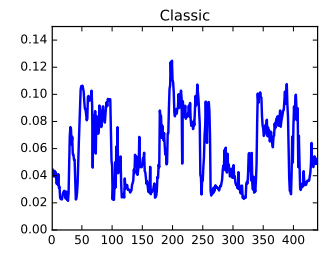

400

Fig. 12. 4D facial shape estimation on 4DMaja-synthetic video: comparison of our video fitting method (ITW-V) with other methods. The results are presented in two ways: a) CEDs of the normalized dense vertex error, b) Plots of the mean normalized vertex error as a function of time (frame index), where all plots share the same vertical axis.

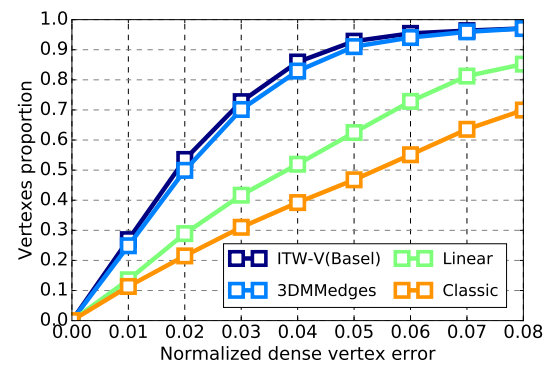

Fig. 13. 3D identity shape estimation on 4DMaja-real video. The results are presented as CEDs of the normalized dense vertex error.

between the vertices of the resulting fits and the ground truth. As presented in Fig. 14 our video fitting outperforms per-frame image fitting by a large margin, validating the merits of our formulation.

Please refer to the Supplementary Material for additional visualisations and self-evaluation experiments.

\section{Conclusion}

We have presented a novel formulation of 3DMMs re-imagined for use in "in-the-wild" conditions. We capitalise on annotated "inthe-wild" facial databases to propose a methodology for learning an "in-the-wild" feature-based texture model suitable for 3DMM fitting on images and videos without having to optimise for illumination parameters. We show that we are able to recover shapes with more detail than is possible using purely landmark-driven

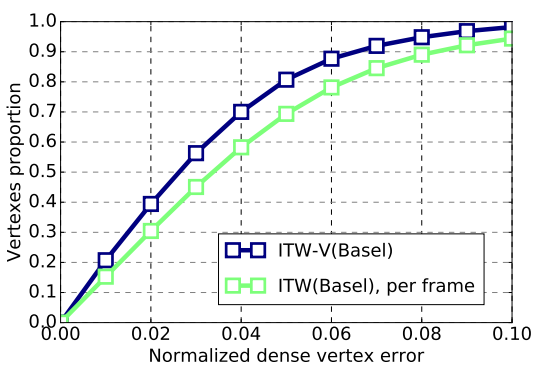

Fig. 14. 4D facial shape estimation on 4DMaja-synthetic video: selfevaluation of our fitting framework. Comparison of our video fitting method (ITW-V(Basel)) with our image fitting method applied per-frame (ITW(Basel), per-frame), i.e. independently on every frame of the video. The results are presented as CEDs of the per-frame mean (over all vertices) normalized dense vertex error.

approaches. Our newly introduced "in-the-wild" benchmarks, KFITW, 3dMDLab \& 4DMaja, permit for the first time a quantitative evaluation of 3D facial reconstruction techniques "in-the-wild" on images and videos, and on these evaluations we demonstrate that our "in-the-wild" formulation is state-of-the-art, outperforming contemporary 3DMM approaches by a considerable margin.

\section{ACKNOWLEDGMENTS}

J. Booth was supported by an EPSRC DTA award. The work of A. Roussos, S. Ploumpis and S. Zafeiriou has been partially funded by the EPSRC Project EP/N007743/1 (FACER2VM). We 
would like to thank George Trigeorgis for his invaluable help in the preparation of this article, and the authors of [71] for kindly providing us with results of their method.

\section{REFERENCES}

[1] V. Jain and E. Learned-Miller, "FDDB: A benchmark for face detection in unconstrained settings," University of Massachusetts, Amherst, Tech. Rep. UM-CS-2010-009, 2010.

[2] S. Zafeiriou, C. Zhang, and Z. Zhang, "A survey on face detection in the wild: past, present and future," CVIU, vol. 138, pp. 1-24, 2015.

[3] X. Xiong and F. De la Torre, "Supervised descent method and its applications to face alignment," in CVPR, 2013, pp. 532-539.

[4] V. Kazemi and J. Sullivan, "One millisecond face alignment with an ensemble of regression trees," in CVPR, 2014, pp. 1867-1874.

[5] A. Asthana, S. Zafeiriou, S. Cheng, and M. Pantic, "Incremental face alignment in the wild," in CVPR, 2014, pp. 1859-1866.

[6] G. Tzimiropoulos and M. Pantic, "Gauss-newton deformable part models for face alignment in-the-wild," in CVPR, 2014, pp. 1851-1858.

[7] S. Zhu, C. Li, C. Change Loy, and X. Tang, "Face alignment by coarseto-fine shape searching," in CVPR, 2015, pp. 4998-5006.

[8] E. Antonakos, J. Alabort-i-Medina, G. Tzimiropoulos, and S. Zafeiriou, "Feature-based lucas-kanade and active appearance models," TIP, vol. 24, no. 9, pp. 2617-2632, September 2015.

[9] E. Antonakos, J. Alabort-i-Medina, and S. Zafeiriou, "Active Pictorial Structures," in CVPR. Boston, MA, USA: IEEE, June 2015, pp. 54355444.

[10] G. Trigeorgis, P. Snape, M. Nicolaou, E. Antonakos, and S. Zafeiriou, "Mnemonic Descent Method: A recurrent process applied for end-to-end face alignment," in CVPR. Las Vegas, NV, USA: IEEE, June 2016.

[11] V. Le, J. Brandt, Z. Lin, L. Bourdev, and T. S. Huang, "Interactive facial feature localization," in ECCV. Springer, 2012, pp. 679-692.

[12] X. Zhu and D. Ramanan, "Face detection, pose estimation, and landmark localization in the wild," in CVPR. IEEE, 2012, pp. 2879-2886.

[13] P. N. Belhumeur, D. W. Jacobs, D. J. Kriegman, and N. Kumar, "Localizing parts of faces using a consensus of exemplars," IEEE T-PAMI, vol. 35, no. 12, pp. 2930-2940, 2013.

[14] C. Sagonas, G. Tzimiropoulos, S. Zafeiriou, and M. Pantic, "300 faces in-the-wild challenge: The first facial landmark localization challenge," in $I C C V$ - $W$, Sydney, Australia, December 2013.

[15] C. Sagonas, E. Antonakos, G. Tzimiropoulos, S. Zafeiriou, and M. Pantic, "300 faces in-the-wild challenge: Database and results," Image and Vision Computing, vol. 47, pp. 3-18, 2016.

[16] C. Cao, Y. Weng, S. Zhou, Y. Tong, and K. Zhou, "Facewarehouse: A 3d facial expression database for visual computing," $T-V C G$, vol. 20, no. 3 , pp. 413-425, 2014.

[17] P. Paysan, R. Knothe, B. Amberg, S. Romdhani, and T. Vetter, "A 3d face model for pose and illumination invariant face recognition," in AVSS. IEEE, 2009, pp. 296-301.

[18] J. Booth, A. Roussos, S. Zafeiriou, A. Ponniah, and D. Dunaway, "A 3d morphable model learnt from 10,000 faces," in CVPR, 2016.

[19] O. Aldrian and W. A. Smith, "Inverse rendering of faces with a $3 \mathrm{~d}$ morphable model," T-PAMI, vol. 35, no. 5, pp. 1080-1093, 2013.

[20] P. Huber, P. Kopp, W. Christmas, M. Rätsch, and J. Kittler, "Real-time $3 \mathrm{~d}$ face fitting and texture fusion on in-the-wild videos," IEEE Signal Processing Letters, vol. 24, no. 4, pp. 437-441, 2017.

[21] N. Dalal and B. Triggs, "Histograms of oriented gradients for human detection," in CVPR, vol. 1. IEEE, 2005, pp. 886-893.

[22] D. G. Lowe, "Object recognition from local scale-invariant features," in ICCV, vol. 2. Ieee, 1999, pp. 1150-1157.

[23] E. Antonakos, J. Alabort-i-Medina, G. Tzimiropoulos, and S. Zafeiriou, "Hog active appearance models," in ICIP. IEEE, 2014, pp. 224-228.

[24] G. Papandreou and P. Maragos, "Adaptive and constrained algorithms for inverse compositional active appearance model fitting," in $C V P R$. IEEE, 2008, pp. 1-8.

[25] G. Tzimiropoulos and M. Pantic, "Optimization problems for fast aam fitting in-the-wild," in ICCV, 2013, pp. 593-600.

[26] J. Alabort-i Medina and S. Zafeiriou, "A unified framework for compositional fitting of active appearance models," IJCV, pp. 1-39, 2016.

[27] J. Alabort-i-Medina, E. Antonakos, J. Booth, P. Snape, and S. Zafeiriou, "Menpo: A comprehensive platform for parametric image alignment and visual deformable models," in ACM ICM, ser. MM '14. New York, NY, USA: ACM, 2014, pp. 679-682.

[28] L. Sirovich and M. Kirby, "Low-dimensional procedure for the characterization of human faces." JOPT, vol. 4, no. 3, pp. 519-524, Mar. 1987.
[29] A. S. Georghiades, P. N. Belhumeur, and D. Kriegman, "From few to many: Illumination cone models for face recognition under variable lighting and pose," TPAMI, vol. 23, no. 6, pp. 643-660, 2001.

[30] R. Basri and D. W. Jacobs, "Lambertian reflectance and linear subspaces," TPAMI, vol. 25, pp. 218-233, Feb. 2003.

[31] M. Turk and A. Pentland, "Eigenfaces for recognition," Journal of cognitive neuroscience, vol. 3, no. 1, pp. 71-86, 1991

[32] R. Ramamoorthi, "Analytic pca construction for theoretical analysis of lighting variability in images of a lambertian object," TPAMI, vol. 24 no. 10, pp. 1322-1333, 2002.

[33] R. Ramamoorthi and P. Hanrahan, "On the relationship between radiance and irradiance: determining the illumination from images of a convex lambertian object," JOPT, vol. 18, no. 10, pp. 2448-2459, 2001.

[34] A. Shashua, "On photometric issues in 3d visual recognition from a single 2d image," IJCV, vol. 21, no. 1-2, pp. 99-122, 1997.

[35] S. R. Marschner, S. H. Westin, E. P. Lafortune, K. E. Torrance, and D. P. Greenberg, "Image-based brdf measurement including human skin," in Eurographics Workshop on Rendering. Springer, 1999, pp. 131-144.

[36] P. Snape and S. Zafeiriou, "Kernel-pca analysis of surface normals for shape-from-shading," in CVPR, 2014, pp. 1059-1066.

[37] P. L. Worthington and E. R. Hancock, "New constraints on data-closeness and needle map consistency for shape-from-shading," T-PAMI, vol. 21, no. 12, pp. 1250-1267, 1999.

[38] W. A. Smith and E. R. Hancock, "Facial shape-from-shading and recognition using principal geodesic analysis and robust statistics," IJCV, vol. 76, no. 1, pp. 71-91, 2008

[39] J. T. Barron and J. Malik, "Shape, illumination, and reflectance from shading," T-PAMI, vol. 37, no. 8, pp. 1670-1687, 2015.

[40] W. A. Smith and E. R. Hancock, "Recovering facial shape using a statistical model of surface normal direction," T-PAMI, vol. 28 , no. 12 , pp. 1914-1930, 2006.

[41] P. Snape, Y. Panagakis, and S. Zafeiriou, "Automatic construction of robust spherical harmonic subspaces," in CVPR, 2015, pp. 91-100.

[42] I. Kemelmacher-Shlizerman, "Internet based morphable model," in ICCV, 2013, pp. 3256-3263.

[43] V. Blanz and T. Vetter, "A morphable model for the synthesis of $3 \mathrm{~d}$ faces," in CGIT. ACM Press/Addison-Wesley Publishing Co., 1999, pp. 187-194.

[44] _ - "Face recognition based on fitting a 3d morphable model," T-PAMI, vol. 25, no. 9, pp. 1063-1074, 2003.

[45] S. Romdhani and T. Vetter, "Efficient, robust and accurate fitting of a 3d morphable model." in ICCV, vol. 3, 2003, pp. 59-66.

[46] B. Amberg, "Editing faces in videos," Ph.D. dissertation, University_of_Basel, 2011.

[47] S. Romdhani and T. Vetter, "Estimating 3d shape and texture using pixel intensity, edges, specular highlights, texture constraints and a prior," in CVPR, vol. 2. IEEE, 2005, pp. 986-993.

[48] J. Thies, M. Zollhofer, M. Stamminger, C. Theobalt, and M. Nießner, "Face2face: Real-time face capture and reenactment of rgb videos," in CVPR, 2016, pp. 2387-2395.

[49] R. Garg, A. Roussos, and L. Agapito, "A variational approach to video registration with subspace constraints," IJCV, vol. 104, no. 3, pp. 286 314, 2013.

[50] — "Dense variational reconstruction of non-rigid surfaces from monocular video," in CVPR, 2013, pp. 1272-1279.

[51] M. Hernandez, T. Hassner, J. Choi, and G. Medioni, "Accurate 3d face reconstruction via prior constrained structure from motion," Computers \& Graphics, vol. 66, pp. 14-22, 2017.

[52] J. Booth, A. Roussos, A. Ponniah, D. Dunaway, and S. Zafeiriou, "Large scale 3d morphable models," International Journal of Computer Vision, vol. 126 , no. $2-4$, pp. 233-254, 2018

[53] S. Cheng, I. Marras, S. Zafeiriou, and M. Pantic, "Statistical non-rigid icp algorithm and its application to 3d face alignment," Image and Vision Computing, 2016

[54] J. B. Kuipers et al., Quaternions and rotation sequences. Princeton university press Princeton, 1999, vol. 66

[55] M. Wheeler and K. Ikeuchi, "Iterative estimation of rotation and translation using the quaternion: School of computer science," 1995.

[56] F. Shang, Y. Liu, J. Cheng, and H. Cheng, "Robust principal component analysis with missing data," in CIKM, ser. CIKM '14. New York, NY, USA: ACM, 2014, pp. 1149-1158.

[57] D. P. Bertsekas, Constrained optimization and Lagrange multiplier methods. Academic press, 2014.

[58] A. Jourabloo and X. Liu, "Large-pose face alignment via cnn-based dense 3d model fitting," in CVPR, 2016.

[59] S. Baker and I. Matthews, "Lucas-kanade 20 years on: A unifying framework," IJCV, vol. 56, no. 3, pp. 221-255, 2004. 
[60] I. Matthews and S. Baker, "Active appearance models revisited," IJCV, vol. 60 , no. 2 , pp. 135-164, 2004

[61] J. Booth, E. Antonakos, S. Ploumpis, G. Trigeorgis, Y. Panagakis, and S. Zafeiriou, "3d face morphable models "in-the-wild"," in CVPR, 2017.

[62] M. Zollhöfer, M. Nießner, S. Izadi, C. Rehmann, C. Zach, M. Fisher, C. Wu, A. Fitzgibbon, C. Loop, C. Theobalt et al., "Real-time non-rigid reconstruction using an rgb-d camera," ACM Transactions on Graphics (TOG), vol. 33, no. 4, p. 156, 2014.

[63] S. Izadi, D. Kim, O. Hilliges, D. Molyneaux, R. Newcombe, P. Kohli, J. Shotton, S. Hodges, D. Freeman, A. Davison et al., "Kinectfusion: realtime $3 \mathrm{~d}$ reconstruction and interaction using a moving depth camera," in UIST. ACM, 2011, pp. 559-568.

[64] R. A. Newcombe, S. Izadi, O. Hilliges, D. Molyneaux, D. Kim, A. J. Davison, P. Kohi, J. Shotton, S. Hodges, and A. Fitzgibbon, "Kinectfusion: Real-time dense surface mapping and tracking," in ISMAR. IEEE, 2011, pp. 127-136.

[65] X. Zhu, Z. Lei, X. Liu, H. Shi, and S. Z. Li, "Face alignment across large poses: A 3d solution," in CVPR, June 2016.

[66] J. Deng, Y. Zhou, S. Cheng, and S. Zafeiriou, "Cascade multi-view hourglass model for robust 3d face alignment," in $F G, 2018$.

[67] G. Chrysos, E. Antonakos, P. Snape, A. Asthana, and S. Zafeiriou, "A comprehensive performance evaluation of deformable face tracking "inthe-wild"," IJCV, 2017.

[68] P. Huber, G. Hu, R. Tena, P. Mortazavian, W. P. Koppen, W. Christmas, M. Rätsch, and J. Kittler, "A multiresolution 3d morphable face model and fitting framework," in VISAPP, 2016.

[69] A. Bas, W. A. Smith, T. Bolkart, and S. Wuhrer, "Fitting a 3d morphable model to edges: A comparison between hard and soft correspondences," in Asian Conference on Computer Vision. Springer, 2016, pp. 377-391.

[70] A. S. Jackson, A. Bulat, V. Argyriou, and G. Tzimiropoulos, "Large pose $3 \mathrm{~d}$ face reconstruction from a single image via direct volumetric cnn regression," International Conference on Computer Vision, 2017.

[71] A. Tewari, M. Zollöfer, H. Kim, P. Garrido, F. Bernard, P. Perez, and T. Christian, "MoFA: Model-based Deep Convolutional Face Autoencoder for Unsupervised Monocular Reconstruction," in The IEEE International Conference on Computer Vision (ICCV), 2017.

[72] S. Zafeiriou, M. Hansen, G. Atkinson, V. Argyriou, M. Petrou, M. Smith, and L. Smith, "The photoface database," in CVPR, June 2011, pp. 132 139.

[73] R. Basri, D. Jacobs, and I. Kemelmacher, "Photometric stereo with general, unknown lighting," IJCV, vol. 72, no. 3, pp. 239-257, 2007.

[74] D. Marr and H. K. Nishihara, "Representation and recognition of the spatial organization of three-dimensional shapes," Royal Society of London B: Biological Sciences, vol. 200, no. 1140, pp. 269-294, 1978.

[75] J. Shen, S. Zafeiriou, G. Chrysos, J. Kossaifi, G. Tzimiropoulos, and M. Pantic, "The first facial landmark tracking in-the-wild challenge: Benchmark and results," in $I C C V$ - $W$, December 2015.

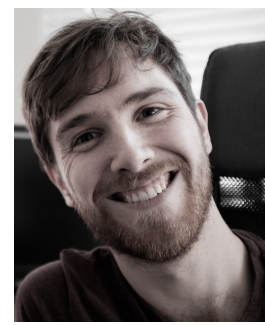

James Booth recently completed his $\mathrm{PhD}$ at Imperial College London under the supervision of Dr. Stefanos Zafeiriou. His thesis covered the construction and application of highly accurate 3D deformable facial models. James was also an honorary member of the Craniofacial Unit at Great Ormond Street Hospital London where he applied his research to advancing the state of the art in craniofacial surgery planning and outcome measurements. He now works at Oculus VR.

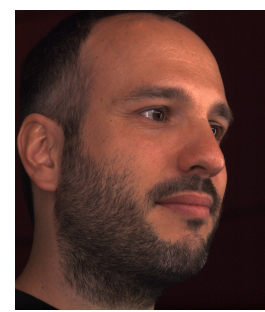

Anastasios Roussos is a Lecturer (equivalent to Assistant Professor) in Computer Science at the University of Exeter, UK. He is also affiliated with the Department of Computing, Imperial College London. Prior to these positions, he has worked as a postdoctoral researcher at University College London (UCL) (2013-2014) and Queen Mary, University of London (20102013). His research specialises in the fields of Computer Vision and Machine Learning.

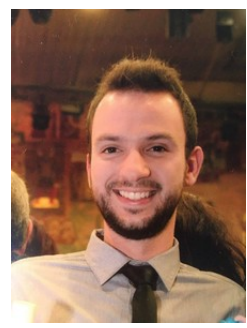

Evangelos Ververas joined the Intelligent Behavior Understanding Group (IBUG) at the Department of Computing, Imperial College London, in October 2016 and is currently working as a PhD Student/Teaching Assistant under the supervision of Dr. Stefanos Zafeiriou. His research focuses on machine learning and computer vision models for $3 \mathrm{D}$ reconstruction and analysis of human faces.

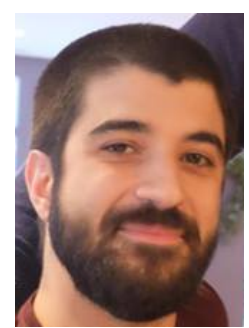

Epameinondas Antonakos is a Computer $\mathrm{Vi}$ sion Applied Scientist at Amazon in Berlin, Germany since February 2017. During 2012-2017, he did his PhD at the Department of Computing, Imperial College London, as part of the Intelligent Behaviour Understanding Group (iBUG) under the supervision of Dr. Stefanos Zafeiriou. His PhD work focused on 2D and 3D deformable models for fitting and tracking of the human face.

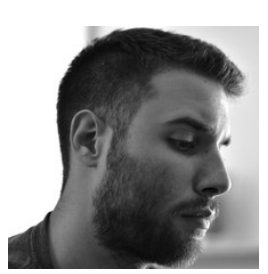

Stylianos Ploumpis is a Research Assistant/PhD student at the Department of Computing at Imperial College London under the supervision of Dr. Stefanos Zafeiriou. His research interests lie in the field of 3D Computer Vision, Pattern Recognition and Machine Learning.

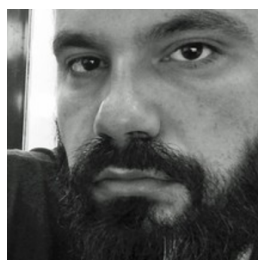

Yannis Panagakis is a Lecturer in Computer Science at Middlesex University London and a Research Fellow at the Department of Computing, Imperial College London. Yannis received various scholarships and awards for his studies and research, including the prestigious MarieCurie Fellowship in 2013.

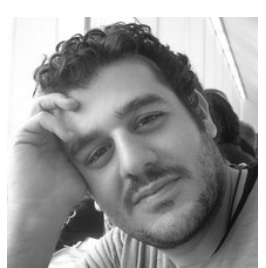

Stefanos Zafeiriou is a Reader in Machine Learning for Computer Vision with the Department of Computing, Imperial College London, and a Distinguishing Research Fellow with University of Oulu under Finish Distinguishing Professor Programme. He was the recipient of a prestigious Junior Research Fellowship from Imperial College London in 2011 to start his own independent research group. He was also the recipient of the President's Medal for Excellence in Research Supervision for 2016. 